\title{
THE QUASI-STEADY-STATE ASSUMPTION: A CASE STUDY IN PERTURBATION *
}

\author{
LEE A. SEGEL $\dagger$ and MARSHALL SLEMROD $\ddagger$
}

\begin{abstract}
The quasi-steady-state assumption (QSSA) of biochemistry is studied as an approximation that is important in itself and also that exemplifies an approach to ODE systems with an initial fast transient. Simple estimates of the two relevant time scales of the underlying problem are made. These estimates lead to a dimensionless formulation containing a different small parameter from that which is customary. Earlier results on the QSSA are surveyed in the context of the new nondimensionalization. It is shown that more comprehensive and simpler error estimates can now be made. Some general methodological hints are drawn from this example.
\end{abstract}

Key words. quasi-steady-state assumption, singular perturbations, scaling, fast transients, Michaelis Menten approximation

\section{AMS(MOS) subject classifications.34, 92}

0. Introduction. An important approximation method, in the study of biochemical kinetics for example but also in many other fields, is an approach variously called the quasi-steady-state assumption (QSSA - see [1]) or the pseudo-steady-state hypothesis (PSSH - see [14]). In its simplest form, the QSSA, as we shall call it here, deals with kinetics described by systems of ordinary differential equations wherein, after an initial fast transient, one (or more) of the dependent variables can be regarded as in steady state with respect to the instantaneous values of the other dependent variables.

Here we examine the QSSA as a case study in approximation. We devote particular attention to the art of choosing suitable dimensionless variables. This allows us to develop several improvements on (the very many) earlier results concerning the QSSA. In particular we are able to determine the "correct" small parameter of the problem, we can extend the QSSA to instances where the classical small parameter is not in fact small, and we can prove a theorem that pays due attention to uniformity of convergence with respect to "nonsmall" parameters. We believe that the spirit of our results will be found of some general utility.

0.1. The enzyme-substrate reaction. Perhaps the most studied example of the QSSA concerns a biochemical reaction wherein an enzyme (concentration $E$ ) reacts reversibly with another chemical (concentration $S$, the substrate) to form an enzymesubstrate complex (concentration $C$ ). With a certain probability per unit time $k_{2}$, the complex irreversibly breaks down into the original enzyme plus an altered substrate molecule called the product (concentration $P$ ). Schematically,

$$
E+S \underset{k_{-1}}{\stackrel{k_{1}}{\rightleftarrows}} C \stackrel{k_{2}}{\longrightarrow} E+P
$$

\footnotetext{
*Received by the editors February 22, 1988; accepted for publication (in revised form) January 24, 1989.

†Department of Applied Mathematics, Weizmann Institute, Rehovot IL-76100, Israel.

$\ddagger$ Center for Mathematical Sciences, University of Wisconsin, Madison, Wisconsin. This research was performed while the author was the Erna and Jakob Michael Visiting Professor at the Weizmann Institute of Science, Rehovot, Israel. His research was supported in part by the Air Force Office of Scientific Research, Air Force Systems Command, under grant 5-28529.
} 
The differential equations that are traditionally taken to govern this situation are

$$
\begin{aligned}
& \frac{d E}{d t}=-k_{1} E S+k_{-1} C+k_{2} C, \\
& \frac{d S}{d t}=-k_{1} E S+k_{-1} C, \\
& \frac{d C}{d t}=k_{1} E S-k_{-1} C-k_{2} C, \\
& \frac{d P}{d t}=k_{2} C .
\end{aligned}
$$

We shall restrict ourselves for now to the (most important) initial conditions

$$
\begin{aligned}
& E(0)=E_{0}, \\
& S(0)=S_{0}, \\
& C(0)=0 \\
& P(0)=0 .
\end{aligned}
$$

Adding (1a) and (1c), and employing (2), we find that

$$
E(t)+C(t)=E_{0}
$$

with which $E$ can be eliminated from (1b) and (1c), yielding the basic mathematical problem

$$
\begin{aligned}
& \frac{d S}{d t}=-k_{1}\left(E_{0}-C\right) S+k_{-1} C, \\
& \frac{d C}{d t}=k_{1}\left(E_{0}-C\right) S-\left(k_{-1}+k_{2}\right) C \\
& S(0)=S_{0} \\
& C(0)=0 .
\end{aligned}
$$

0.2. The quasi-steady-state assumption. Under certain circumstances, the QSSA can be used to simplify the system (3). This simplification and its consequences are generally studied in elementary biochemistry courses. One excellent text [11] proceeds as follows.

It is first pointed out that the experimental measurements to be made generally are performed after a relatively short pre-steady-state period but before the substrate concentration decays appreciably. It is then stated that "if the reaction rate measured is approximately constant over the time interval concerned" then the complex concentration $C$ is approximately constant. (By "reaction rate" is meant the rate of product formation, so that the approximate constancy of $C$ follows from (1d).) If $C$ is approximately constant then $d C / d t \approx 0$. With this we can solve $(3 \mathrm{~b})$ for $C$ in terms of $S$, yielding

$$
C=\frac{E_{0} S}{\left(K_{m}+S\right)}
$$

where

$$
K_{m} \equiv \frac{\left(k_{-1}+k_{2}\right)}{k_{1}} .
$$


Under what circumstances can we assume that $d C / d t \approx 0$ ? A typical assertion in this regard, from another excellent biochemistry text [19], is that "if the concentration of $S$ is high enough, the free enzyme $E$ will immediately combine with another molecule of $S$. Under these conditions a steady state is achieved in which the enzyme is always saturated with its substrate." This quotation correctly implies that the approximation $d C / d t \approx 0$ can be valid for a considerable period of time. When this is the case, upon substituting (4) into the substrate equation (3a) we obtain

$$
\frac{d S}{d t}=-\frac{k_{2} E_{0} S}{K_{m}+S}
$$

Presumably the substrate concentration does not change appreciably during the brief pre-steady-state period, so that to (5a) we may adjoin the initial condition

$$
S(0)=S_{0}
$$

Equations (5a) and (5b) are the essence of the quasi-steady-state assumption (QSSA), so called because in a true steady state $C$ would be constant while here $C$ changes but in such a way as instantaneously to be in steady state with the present value of the substrate concentration.

0.3. The singular perturbation approach. Theoreticians have long been aware of the fact that the QSSA can be obtained from the well-studied singular perturbation theory for equations of the form

$$
\frac{d s}{d T}=f(c, s), \quad \epsilon \frac{d c}{d T}=g(c, s), \quad 0<\epsilon \ll 1 .
$$

Earlier a number of authors obtained such equations by changes of variables. Typical is the approach in the classical paper of Heineken, Tsuchiya, and Aris [14] who write, "let us make (our equations) dimensionless by taking

$$
\begin{aligned}
& y=\frac{S}{S_{0}}, \\
& z=\frac{C}{E_{0}}, \\
& h=k_{1} E_{0} t . "
\end{aligned}
$$

Upon substitution into (3a) and (3b) these variables give the "outer" equations

$$
\begin{aligned}
& \frac{d y}{d h}=-y+(y+\mu-\lambda) z \\
& \epsilon_{h} \frac{d z}{d h}=y-(y+\mu) z .
\end{aligned}
$$

Here

$$
\epsilon_{h}=\frac{E_{0}}{S_{0}}, \quad \mu=\frac{K_{m}}{S_{0}}, \quad \lambda=\frac{k_{2}}{k_{1} S_{0}} .
$$

With the standard change to a fast time variable $H$ via

$$
H=\frac{h}{\epsilon_{h}}
$$


we obtain the "inner equations"

$$
\frac{d y}{d H}=\epsilon_{h}[-y+(y+\mu-\lambda) z], \quad \frac{d z}{d H}=-y-(y+\mu) z
$$

What we shall term the standard dimensionless variables (7) have been introduced without motivation by many authors, not only by Heineken, Tsuchiya, and Aris [14] but also for example in the reviews by Hahn [13] and Klonowski [16] and in the biomathematical texts by Rubinow [28], Murray [22] and Britton [3]. In our view it is unfortunate that the variable change (7) is unmotivated, for this change constitutes the crucial step of the analysis. Moreover, the variables (7) lead to a small parameter $\epsilon_{h} \equiv E_{0} / S_{0}$, but it is a central result of this paper that the small parameter should be taken to be $\epsilon \equiv E_{0} /\left(S_{0}+K_{m}\right)$.

For at least 25 years, the QSSA has been analyzed by theoretical chemists and applied mathematicians (including one of the present authors). That applied mathematicians can profit from a reexamination of this relatively simple problem is attested to by the fact that only now have appropriately broad conditions been discovered for the validity of the QSSA. Not surprisingly in view of the slow progress, fundamental issues of the applied mathematician's trade are involved in proper treatment of the QSSA. Chief among these is the choice of appropriately nondimensionalized variables, for this is the key to an efficient analysis.

1. Choosing time scales: a first analysis of the QSSA. Central to further developments is the selection of appropriate timescales. These will be the basis for deriving necessary conditions for the validity of the QSSA, and also for choosing suitable dimensionless variables.

1.1. Scaling. Concepts of scaling [30], $[20, \S 6.3]$ provide an essential background to our discussion. These are the essential points.

(i) The scale of a dependent variable is a combination of parameters, with the appropriate dimension, that provides an estimate of the variable's maximum order of magnitude.

(ii) The scale of an independent variable is a combination of parameters, with the appropriate dimension, that estimates the range of the independent variable over which there is a significant change in the dependent variables.

(iii) To obtain scaled dimensionless variables dimensional variables are divided by their scales.

(iv) It follows from the definitions that scaled dimensionless variables are of unit order of magnitude. Hence the relative magnitudes of the various terms in scaled dimensionless equations are correctly provided by the dimensionless parameters that appear. This is a decisive help in devising approximation methods.

(v) It may be necessary to choose different scales in different domains of the independent variable. If so, the appropriate approximation methods are of singular perturbation type.

(vi) Different scalings are appropriate in different domains of parameter space.

(vii) Scaling requires prior knowledge of the solution. This can be provided by experiments, "physical intuition," and/or numerical analyses of special cases.

1.2. Scaling and the QSSA. Employing a biophysical point of view, we derive conditions that seem necessary for the validity of the QSSA. As will be seen, these 
conditions are essentially consistency checks. As a key step in our derivation we shall estimate the duration $t_{C}$ of the pre-steady-state period (i.e., we estimate the fast timescale). Assuming that the QSSA is valid after the pre-steady-state period, we shall then estimate the slow timescale $t_{S}$ for the decay of substrate.

To estimate $t_{C}$ we make the approximation $S \approx S_{0}$ in (3b) (compare (5b)). The resulting equation is readily solved, yielding

$$
C(t)=\bar{C}[1-\exp (-k t)]
$$

where (compare (4a))

$$
\begin{aligned}
& \bar{C} \equiv \frac{E_{0} S_{0}}{K_{m}+S_{0}}, \\
& k \equiv k_{1}\left(S_{0}+K_{m}\right) .
\end{aligned}
$$

As a consequence we take

$$
t_{C}=k^{-1}
$$

To estimate $t_{S}$ we employ one of the useful ways to characterize a timescale $[31$, p. 56]

$$
t_{S}=\left(S_{\max }-S_{\min }\right) /\left|\frac{d S}{d t}\right|_{\max } .
$$

To find the maximum value of $d S / d t$ for the period after the initial transient, we substitute $S=S_{0}$ into (5a). It follows at once that

$$
t_{S}=\frac{K_{m}+S_{0}}{k_{2} E_{0}}
$$

An essential feature of the QSSA is that the duration of the pre-steady-state period is much shorter than the characteristic time for substrate change. A necessary condition is thus $t_{C} \ll t_{S}$, i.e.,

$$
\eta \ll(1+\kappa)(1+\sigma)^{2} .
$$

Here we have employed the dimensionless parameters

$$
\sigma \equiv \frac{S_{0}}{K_{m}}, \quad \eta \equiv \frac{E_{0}}{K_{m}}, \quad \kappa \equiv \frac{k_{-1}}{k_{2}} .
$$

Initial condition (5b) is appropriate to the QSSA only if there is a negligible relative change $\left|\Delta S / S_{0}\right|$ in the substrate concentration during the pre-steady state. We estimate $\Delta S / S_{0}$ by

$$
\left|\frac{\Delta S}{S_{0}}\right| \approx \frac{1}{S_{0}}\left|\frac{d S}{d t}\right|_{\max } \cdot t_{C}
$$

Employing (3a) with $C=0$ to determine $|d S / d t|_{\max }$ (in contrast with the derivation of (15)) we find the additional necessary condition

$$
\left|\frac{\Delta S}{S_{0}}\right| \approx \frac{E_{0}}{K_{m}+S_{0}} \ll 1
$$


In dimensionless terms this condition is

$$
\eta \ll 1+\sigma
$$

Note that our new condition (19) is stronger than (16), i.e., (19) implies (16). (See Appendix 1 for further remarks concerning (19).) Note also that a "nominee" for the correct dimensionless parameter of the problem is now apparent, namely, $\epsilon \equiv$ $E_{0} /\left(K_{m}+S_{0}\right) \equiv \eta /(1+\sigma)$.

2. The scaled dimensionless equations, singular perturbations and estimates. Vital for the derivation of the necessary conditions (16) and (19) was our estimation of the two timescales of our problem, the duration $t_{C}$ of the presteady-state period, and the duration $t_{S}$ of the period during which the substrate $S$ is converted to product according to the QSSA. Knowledge of these scales is precisely what is required to choose suitable dimensionless independent variables, a prerequisite to further analysis of the QSSA.

2.1. The scaled equations. In the pre-steady state, it is now clear that time should be scaled by $t_{C}$. The QSSA assumes that in this state $S \approx S_{0}$; this gives the scale for $S$ and also indicates that the complex concentration $C$ will be well estimated by its steady-state value $\bar{C}$ of $(12 \mathrm{~b})$. We thus introduce the scaled dimensionless variables

$$
\begin{aligned}
s & \equiv \frac{S}{S_{0}}, \\
c & \equiv \frac{C}{\bar{C}}, \\
\tau & \equiv \frac{t}{t_{C}},
\end{aligned}
$$

with which the governing equations (3) become

$$
\begin{aligned}
& \frac{d s}{d \tau}=\epsilon\left[-s+\frac{\sigma}{\sigma+1} c s+\frac{\kappa(\kappa+1)^{-1}}{\sigma+1} c\right] \\
& \frac{d c}{d \tau}=s-\frac{\sigma}{\sigma+1} c s-\frac{1}{\sigma+1} c, \\
& s(0)=1 \\
& c(0)=0 .
\end{aligned}
$$

Here, as suggested by (18b) and (19), the small parameter $\epsilon$ is defined by

$$
\epsilon \equiv \frac{\eta}{1+\sigma} \equiv \frac{E_{0}}{K_{m}+S_{0}}
$$

After the pre-steady state, the QSSA is expected to hold. The scaling of the dependent variables remains appropriate but $t_{S}$ is now the correct timescale. We thus introduce a new dimensionless scaled time $T$ by

$$
T \equiv \frac{t}{t_{S}}
$$


Retaining $s$ and $c$ for substrate and complex concentrations as functions of $T$, we note that the governing differential equations now become

$$
\begin{aligned}
& \frac{d s}{d T}=(\kappa+1)(\sigma+1)\left[-s+\frac{\sigma}{\sigma+1} c s+\frac{\kappa(\kappa+1)^{-1}}{\sigma+1} c\right] \\
& \epsilon \frac{d c}{d T}=(\kappa+1)(\sigma+1)\left[s-\frac{\sigma}{\sigma+1} c s-\frac{1}{\sigma+1} c\right]
\end{aligned}
$$

Our problem is now in the form where for $0<\epsilon \ll 1$ approximate solutions can be obtained by standard methods of singular perturbation theory. A solution of (21) is sought of the form

$$
s(\tau)=s^{(0)}(\tau)+\epsilon s^{(1)}(\tau)+\cdots, \quad c(\tau)=c^{(0)}(\tau)+\epsilon c^{(1)}(\tau)+\cdots .
$$

In particular we find that

$$
s^{(0)}(\tau) \equiv 1, \quad c^{(0)}(\tau)=1-e^{-\tau} .
$$

The solution of (24) is assumed to take the form

$$
s(T)=s_{0}(T)+\epsilon s_{1}(T)+\cdots, \quad c(T)=c_{0}(T)+\epsilon c_{1}(T)+\cdots .
$$

Singular perturbation theory supplies matching conditions that fix otherwise unspecified constants. Lin and Segel [20] work through the problem in detail; although they employ different variables, their solution is valid here mutatis mutandis. In particular, it is found at once from (24) that

$$
\begin{aligned}
& c_{0}=\frac{(\sigma+1) s_{0}}{\sigma s_{0}+1} \\
& \frac{d s_{0}}{d T}=-\frac{(\sigma+1) s_{0}}{\sigma s_{0}+1} .
\end{aligned}
$$

The first correction terms in the "outer" solution (27) are found to be

$$
\begin{aligned}
& c_{1}=\frac{\sigma(1+\sigma)}{\left(1+\sigma s_{0}\right)^{3}}\left[\frac{2 s_{0}}{(1+\kappa)\left(1+\sigma s_{0}\right)}-s_{0}+\frac{s_{0} \kappa}{1+\kappa} \ln \left(\frac{1+\sigma s_{0}}{(1+\sigma) s_{0}}\right)\right], \\
& s_{1}=\frac{(1+\sigma) s_{0}}{1+\sigma s_{0}}\left[\frac{\kappa}{1+\kappa} \ln \frac{1+\sigma s_{0}}{(1+\sigma) s_{0}}-\frac{\kappa(1+\kappa)^{-1}+\sigma s_{0}}{1+\sigma s_{0}}\right] .
\end{aligned}
$$

These expressions are useful for performing formal estimates of the errors in the QSSA. Of particular utility are estimates of errors in the complex concentration. This is because the theoretical results are most often compared with the rate of product formation $d P / d t$, which by (1d) is equal to $k_{2} C$.

2.2. Error estimates. Since $C \leq E_{0}$ by $(2 \mathrm{e})$, we shall estimate the ratio of the error in the actual product formation rate, divided by the largest possible product formation rate, by

$$
\delta_{R} \equiv\left|\frac{k_{2}\left(C-C_{s s}\right)}{k_{2} E_{0}}\right| \approx\left|\frac{\bar{C} c_{1}}{E_{0}}\right|=\left|\frac{\sigma \epsilon c_{1}}{\sigma+1}\right|
$$


(In (31), $C_{s s}$ denotes the steady-state value (4a) for complex.) In addition, we estimate the relative error:

$$
\left|\frac{\Delta C}{C}\right| \approx\left|\frac{\epsilon c_{1}}{c_{0}}\right|
$$

By far the most important situation biologically is when the normalized substrate concentration has not decreased too far from its initial value of unity, for as we have mentioned, it is under these circumstances that most measurements are made. Setting $s_{0}=1$ in (29), we obtain

$$
\delta_{R} \approx \frac{\epsilon \sigma^{2}}{(1+\sigma)^{3}}, \quad\left|\frac{\Delta C}{C}\right| \approx \frac{\epsilon}{1+\sigma} \quad \text { when } s_{0} \approx 1 .
$$

In (33) we have omitted the factor $2(1+\kappa)^{-1}(1+\sigma)^{-1}-1$, since its modulus is less than unity.

As $t$ increases and $s_{0}$ falls noticeably below unity, (29) can serve as the basis for an "exact" error estimate, found by maximizing the right-hand sides of $\delta_{R}$ or $\Delta C / C$ as functions of $s_{0}$. We wish to obtain simpler estimates, admitting the cost of some loss in accuracy. To this end, we consider (29) in three situations: (a) $\sigma s_{0} \gg 1$, (b) $\sigma s_{0}=1$, (c) $\sigma s_{0} \ll 1$. In all cases we can write

$$
\text { error } \sim f \epsilon(1+\sigma) \equiv \frac{f E_{0}}{K_{m}}
$$

TABLE 1

At later times estimated error in complex concentration is $f \epsilon(1+\sigma)$ where $f$ is given below.

\begin{tabular}{ccc}
\hline $\begin{array}{c}\text { Approx. substrate } \\
\text { concentration } s_{0}\end{array}$ & "Absolute" error $\delta_{R} \sim\left|\epsilon c_{1}\right|$ & Relative error $\approx\left|\epsilon c_{1} / c_{0}\right|$ \\
\hline $1 \geq s_{0} \gg \sigma^{-1}$ & $f \ll 1$ & $f \ll 1$ \\
$s_{0}=\sigma^{-1}$ & $f=\frac{1}{8} \frac{\kappa}{1+\kappa}\left|-1+\ln \frac{2 \sigma}{1+\sigma}\right|$ & $f=\frac{1}{4} \frac{\kappa}{1+\kappa}\left|-1+\ln \frac{2 \sigma}{(1+\sigma) s_{0}}\right|$ \\
$s_{0} \ll \sigma^{-1}$ & $f=\sigma s_{0}\left|\frac{1-\kappa}{1+\kappa}+\frac{\kappa}{1+\kappa} \ln \frac{1}{(1+\sigma) s_{0}}\right|$ & $f=\left|\frac{1-\kappa}{1+\kappa}+\frac{\kappa}{1+\kappa} \ln \frac{1}{(1+\sigma) s_{0}}\right|$ \\
\hline
\end{tabular}

Estimates of $f$ are given in Table 1 . There is negligible error when $s_{0}$ is such that case (a) obtains, so that this case need not be considered further. The relative error could be large when $\sigma s_{0} \ll 1$ and $\sigma=O(1)$ if $\left|\ln \left(\sigma s_{0}\right)\right|$ is comparable to $\epsilon^{-1}$. But this would occur only when $s_{0} \ll 1$, i.e., when the reaction has proceeded so far that the results are no longer of interest. If $\sigma$ is large then $\left|\ln \left(\sigma s_{0}\right)\right|$ need not be large to produce inaccuracy but then the restriction $s_{0} \ll \sigma^{-1}$ applies to even less interesting conditions. It also should be borne in mind that the $P \rightarrow S$ back reaction, omitted in (1), becomes important when most substrate has been transformed into product. This is a strong reason for ignoring results that formally apply when $s_{0}$ is small.

When $s=\sigma^{-1}$, errors can be large if $\sigma=O\left(\epsilon^{-1}\right)$. Once again, possible significant errors enter only when $s_{0}$ is very small (order $\epsilon$ ). Thus our estimates indicate that for all practical purposes the QSSA is indeed valid when $\epsilon \ll 1$. The estimates (33) show that the error is particularly small when $\sigma \equiv S_{0} / K_{m} \gg 1$. 
3. Mathematical scaling. Some of those who are exposed to the biophysical arguments that lead to dimensionless scaled variables such as (20) and (23) object that there must be a "more mathematical" way to arrive at such variables. In an effort to examine this assertion let us introduce arbitrary scales $\alpha, \beta$, and $\gamma$ and in as "mathematical" a way as possible let us examine what restrictions on these scales will lead to an appropriate problem of singular perturbation type.

Upon adopting the change of variables

$$
S=\alpha s, \quad C=\beta c, \quad T=\gamma t
$$

we find that the governing equations (3a) and (3b) become

$$
\begin{aligned}
& \frac{\alpha}{\gamma} \frac{d s}{d T}=-k_{1} E_{0} \alpha s+k_{1} \beta \alpha c s+k_{-1} \beta c \\
& \frac{\beta}{\gamma} \frac{d c}{d T}=k_{1} E_{0} \alpha s-\left(k_{1} \beta \alpha s+k_{-1} \beta+k_{2} \beta\right) c .
\end{aligned}
$$

3.1. Conventional balancing. Perhaps the most usual way to proceed is via the determination of pairwise balances. One might argue as follows.

(i) The heart of the QSSA is the assumption that the $d c / d T$ term in $(35 \mathrm{~b})$ is negligible.

(ii) If (i) holds there must be a balance between the positive term and one of the negative terms on the right side of $(35 \mathrm{~b})$. If we select the first of the negative terms for balance we obtain (if $c=O(1)$ ) the condition

$$
k_{1} E_{0} \alpha=k_{1} \beta \alpha .
$$

(iii) Since the reaction is irreversible, the substrate concentration must continually decrease. Thus the left side of (35a) must be balanced by the negative term on the right side. This gives

$$
\frac{\alpha}{\gamma}=k_{1} E_{0} \alpha
$$

(iv) The two conditions (36) and (37) yield

$$
\beta=E_{0}, \quad \gamma=\frac{1}{k_{1} E_{0}} .
$$

In addition, elementary intuition indicates that $\alpha=S_{0}$ is the proper scale for the substrate. We thus recover the particular "outer" scaling (7) of [14].

(v) The "inner" timescale is obtained by dividing the outer scale $\gamma$ by the small parameter $\epsilon_{h}$, in order that the $d c / d T$ term be retained.

Two objections may be raised to the procedure (i)-(v). The scaling was partly motivated by mathematical considerations, but physical reasoning, which we are trying to avoid at present, also entered. In addition, although legitimate balances were chosen, other possibilities remain, and indeed the "correct" scaling was not obtained.

3.2. Minimum simplification. Both the above objections are met if we adopt the Principle of Minimum Simplification, as enunciated by Kruskal [17]. (It is unfortunate that this principle, and much other wisdom concerning "asymptotology" 
was promulgated in a relatively inaccessible publication. But see [10] for considerable further exposition of closely related ideas.) Kruskal states that

The basic way systems simplify is by the neglect of terms... but no term should be neglected without a good reason... [Thus] the most widely applicable and hence most informative ordering is that which simplifies the least, maintaining a maximal set of comparable terms. Quite often there is more than one possible maximal set of terms. . . Each maximal set corresponds to different asymptotic behavior.

Let us apply the Principle of Minimal Simplification to the QSSA. As is usual with valuable general principles, blind application might be unsuccessful, but the situation is easily remedied by applying "the inalienable right to think while using any technique" [5].

Although it is customary to regard the initial layer as an adjunct to the QSSA, added for completeness, we should first scale the initial variables, because the initial equations are supplemented by the initial conditions (3c) and (3d). (Nonzero initial conditions give information about the magnitudes of the variables involved, at least for a while.) Nothing can be learned about the scaling from (3d) but from (3c) it follows at once that $\alpha=S_{0}$.

The scale $\beta$ for the complex can be chosen next, independently of $\gamma$, if we rewrite (35b) slightly, using the finding that $\alpha=S_{0}$, as

$$
\gamma^{-1} \frac{d c}{d T}=\frac{k_{1} E_{0} S_{0}}{\beta} s-\left(k_{1} S_{0} s+k_{-1}+k_{2}\right) c .
$$

In the spirit of the Principle of Minimal Simplification we attempt to ensure that all the terms on the right side of (38) are of the same magnitude. In doing so we employ the supposition that $s$ and $c$, as putative scaled variables, are $O(1)$. In fact, for scaling purposes we set $s=c=1$, obtaining

$$
\frac{k_{1} E_{0} S_{0}}{\beta}=k_{1} S_{0}+k_{-1}+k_{2}, \quad \text { i.e., } \beta=\frac{E_{0} S_{0}}{\left(S_{0}+K_{m}\right)} \text {. }
$$

This yields the "correct" scaling (20b). Now $\gamma$ is determined by requiring that the left side of (38) is of same magnitude as the right side, i.e.,

$$
\gamma=\frac{\beta}{k_{1} E_{0} S_{0}}=\left[k_{1}\left(S_{0}+K_{m}\right)\right]^{-1}
$$

showing that the timescale in the pre-steady state, or "inner" layer, is indeed precisely $t_{C}$.

We now proceed to the outer layer. We accept the normal assumption that only the timescale need be changed. In the outer layer the left side of (35a) must be retained. We thus balance the left side with all the terms on the right side of $(35 \mathrm{a})$, again setting $c=s=1$. This gives

$$
\gamma^{-1}=-k_{1} E_{0}+k_{1} \beta+k_{-1} \beta S_{0}^{-1}, \quad \text { i.e., } \gamma=t_{S} .
$$

We note that

$$
\frac{t_{C}}{t_{S}}=\frac{\epsilon}{(1+\kappa)(1+\sigma)} .
$$


That is, the ratio of the inner timescale to the outer is of order $\epsilon$. Note that this ratio is not simply equal to $\epsilon$ as is frequently assumed (for example, in positing the "standard" fast time variable (10)).

In summary, we were able to accomplish an almost automatic scaling of our problem (3) by applying the Principle of Minimal Simplification and setting the scaled dependent variables equal to unity. The results that we obtained were identical to those deduced from our original "physical" approach to scaling. This identity is somewhat surprising; one would expect in general that different approaches would give results that differed by inessential factors of order unity. Moreover there is no a priori reason why application of minimal simplification should lead to scaled variables. But perhaps this was to have been expected, since the procedure gave a unique result when scaling $(c=s=1)$ was assumed.

4. Further developments. This section (i) presents numerical evidence that supports our earlier reasoning, (ii) shows that the results remain valid for general initial conditions, (iii) provides further insight into the necessary condition $t_{C} \ll t_{S}$, and (iv) formulates an extended QSSA for instances wherein $t_{C} \ll t_{S}$ but nonnegligible amounts of substrate $S$ are consumed during the fast transient.

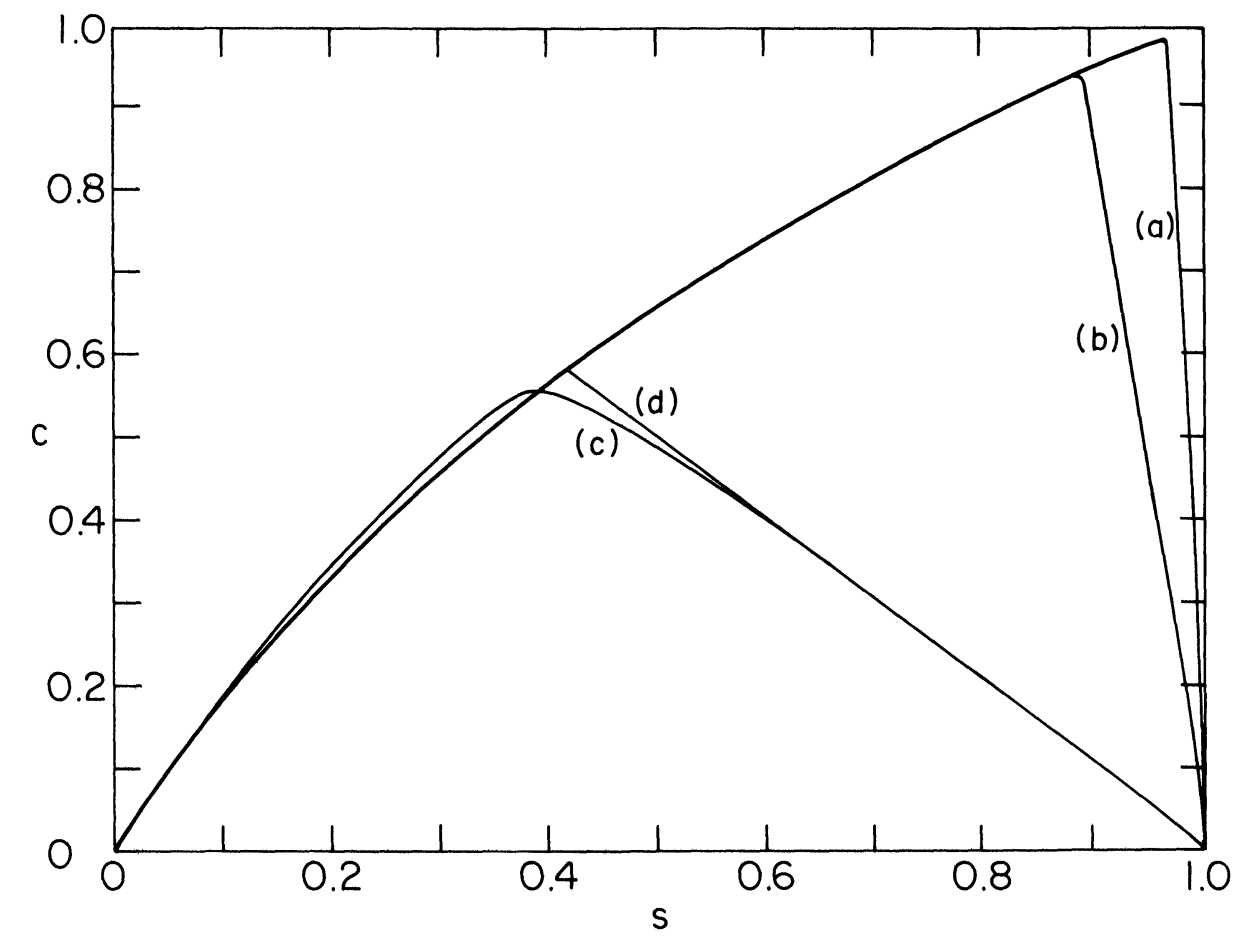

FIG. 1. Phase plane plots of the solutions to (24a), (24b) with initial conditions $s(0)=1$, $c(0)=0, \sigma=1$. For cases $(\mathrm{a})$ and $(\mathrm{b}),(\kappa=10, \epsilon=0.01$, and $\epsilon=0.1)$ the trajectory rapidly (in $T \approx 0.1$ or less see Table 2 ) reaches the QSSA nullcline $c=2 s /(s+1)$ (heavy line). There is little substrate diminution during this period. For case $(\mathrm{c})(\epsilon=1, \kappa=10)$ the substrate diminishes appreciably during the fast transient, but (since (16) holds, i.e., $\epsilon \ll(1+\kappa)(1+\sigma)$ ) the QSSA nullcline eventually serves as an adequate approximation to the solution. The "eventual" QSSA approximation is excellent if $\kappa$ is elevated to 100 , as in (d). 
4.1. Numerical simulations. Numerical simulations provide a check on our reasoning. Figure 1 presents some $s-c$ phase plane plots of solutions to equations (24a), (24b) with initial conditions

$$
s(0)=1, \quad c(0)=0 .
$$

Recall that these dimensionless equations have a substrate scale $S_{0}$, a complex scale $\bar{C}$, and a timescale $t_{S}$. Thus $s$ will decrease from an initial value of unity while $c$ should rapidly attain a value of order unity and then decrease to zero.

Figure 1 indeed shows that for $\epsilon=0.01(\epsilon=0.1)$ there are very small (fairly small) decreases in substrate concentration during the fast transient period wherein $c$ rises to close to unity. As anticipated, since $\epsilon$ is small, the solution then traces out the nullcline of the QSSA. When $\epsilon$ is unity, the QSSA fails, as expected, in that there is a sizable decrease of substrate concentration during the fast transient. Nonetheless, the solution ultimately follows the QSSA nullcline. We shall comment on this shortly.

TABLE 2

Calculated and predicted properties of the fast transient, obtained by integrating system (24) with initial conditions $s(0)=1, c(0)=0$, for $\sigma=1$. The values of $s$ and $c$ at the end of the fast transient, $s^{(i)}$ and $c^{(m)}$, are compared with the approximations of (51) and (52). The cases (a)-(d) correspond to the curves in Fig. 1.

\begin{tabular}{llllllll}
\hline Case & $\epsilon$ & $\kappa$ & $t_{C} / t_{S}$ & $\begin{array}{c}\text { Time to } c^{(m)} \\
\left.\text { (fraction of } t_{S}\right)\end{array}$ & $\begin{array}{l}c^{(m)} \text { calculated } \\
\text { (predicted) }\end{array}$ & $\begin{array}{l}s^{(i)} \text { calculated } \\
\text { (predicted) }\end{array}$ & $\begin{array}{l}\text { Fraction } c^{(m)} \\
\text { reached in } t_{C}\end{array}$ \\
\hline (a) & .01 & 10 & 0.00045 & 0.004 & $0.993(0.995)$ & $0.99(0.99)$ & 0.63 \\
(b) & .1 & 10 & 0.0045 & 0.03 & $0.94(0.95)$ & $0.88(0.905)$ & 0.65 \\
(c) & 1 & 10 & 0.045 & 0.13 & $0.56(0.6)$ & $0.39(0.4)$ & 0.82 \\
(d) & 1 & 100 & 0.0045 & 0.022 & $0.58(0.6)$ & $0.41(0.4)$ & 0.78 \\
\hline
\end{tabular}

TABLE 3

Fraction of product formed as a function of time $T=t / t_{S}$ measured in units of the substrate timescale $t_{S} . \sigma \equiv S_{0} / K_{m}=1$. (Cases (a) -(d) correspond to Fig. 1.)

\begin{tabular}{lllcc}
\hline Case & $\epsilon$ & $\kappa$ & $T=1$ & $T=2$ \\
\hline (a) & 0.01 & 10 & 0.72 & 0.95 \\
(b) & 0.1 & 10 & 0.69 & 0.93 \\
(c) & 1.0 & 10 & 0.47 & 0.71 \\
(d) & 1.0 & 100 & 0.46 & 0.71 \\
\hline
\end{tabular}

If our scaling is correct, the overall decay of $s$ and $c$ should take a time of order unity, while (42) implies that the fast build-up of $c$ should be characterized by a timescale of order $\epsilon /(1+\kappa)(1+\sigma)$. Tables 2 and 3 show that our expectations are borne out. Note from Table 2 that although the timescale of the fast transient is indeed verified to be $t_{C}$, the time for $c$ actually to attain its maximum value $c_{m}$ is typically an order of magnitude longer -- since the change in complex concentration is already taking place more slowly when $c \approx c_{m}$. One of our major points has been the claim that the QSSA should be valid even if the "traditional" small parameter $E_{0} / S_{0}$ 
is of order unity, provided that $\epsilon \equiv E_{0} /\left(S_{0}+K_{m}\right) \ll 1$. Some numerical evidence that supports this claim is presented in Fig. 2.

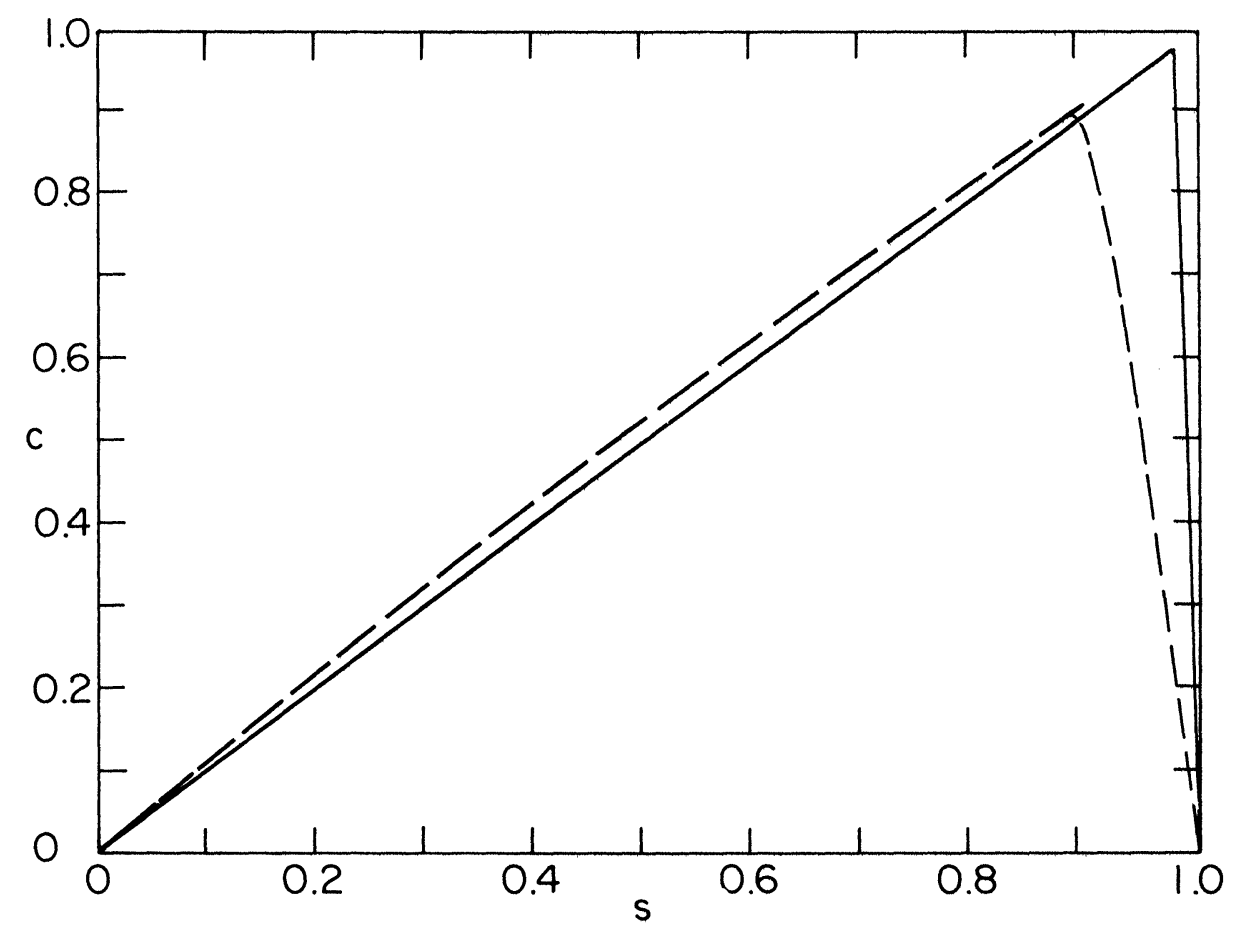

Fig. 2. Plots as in Fig. 1 for $\epsilon_{h} \equiv E_{0} / S_{0}=1, \kappa=10$. Solid curve: $\epsilon=1 / 91, \sigma=1 / 90$. Dashed curve: $\epsilon=1 / 10, \sigma=1 / 9$. The corresponding QSSA graphs are drawn as heavy lines. Note that, as predicted, the QSSA is a good approximation when $\epsilon$ is small, even when $E_{0}=S_{0}$. Figs. 1 and 2 were drawn by the BIOGRAPH program [25].

4.2. General initial conditions. We can easily generalize our considerations to arbitrary initial conditions, wherein

$$
\begin{aligned}
& C(0)=C_{0}, \\
& P(0)=P_{0} .
\end{aligned}
$$

Only (43a) effects our principal developments, for the product concentration can be calculated separately. The conservation law (2e) and the governing equations (3a), (3b) must be modified by the substitution

$$
\begin{aligned}
& E_{0} \rightarrow E_{T}, \\
& E_{T} \equiv E_{0}+C_{0},
\end{aligned}
$$

with corresponding modifications in succeeding formulae. In particular the timescale $t_{C}$ remains as in (13), independent of $C_{0}$, but (44a) modifies both $t_{S}$ and estimate (18b) for $\left|\Delta S / S_{0}\right|$. The fundamental small parameter is thus generalized to $\epsilon \equiv$ $E_{T} /\left(K_{m}+S_{0}\right)$.

4.3. Another view of the requirement $t_{C} \ll t_{S}$. Further insight into the nature of the QSSA can be obtained by re-examining the condition (that led to (16)) that the complex timescale is small with respect to the substrate timescale. 
Consider any substrate level $S$. The timescale $t_{C}$, the intrinsic $C$ timescale with respect to $S$ (to give $t_{C}$ a precise description) estimates the time it takes for $C$ to attain a steady state if $S$ is held fixed. From (12c) and (13)

$$
t_{C}=\left[k_{1}\left(S+K_{m}\right)\right]^{-1} \text {. }
$$

For the QSSA to be consistent, $t_{C}$ must be small compared to $t_{S}$, the timescale for changes in $S$, assuming the QSSA holds. Indeed, if in a timescale $t_{C}$ a process reaches a steady state with a constant environment then that process should be in a quasisteady-state with a changing environment provided the environmental change occurs on a timescale that is long compared to $t_{C}$.

To estimate $t_{S}$ we employ the characterization, slightly different from (14),

$$
t_{S} \approx\left|\frac{S}{d S / d t}\right|
$$

(Equation (45b) makes the reasonable assertion that the time for significant change in a variable with the current value $S(t)$ can be determined by calculating how long it would take to reduce $S$ to zero at the present rate of decrease, $d S(t) / d t$.) After the fast transient, the QSSA equation (5a) for $d S / d t$ permits the following deduction from (45b):

$$
t_{S} \approx \frac{K_{m}+S}{k_{2} E_{0}}
$$

Given (45a) and (45c) we can write

$$
\frac{t_{C}}{t_{S}}=\frac{E_{0}}{K_{m}+S} \frac{1}{1+\kappa} \frac{1}{1+\left(S / K_{m}\right)} .
$$

If the solution trajectory reaches the QSSA nullcline $d C / d t=0$, i.e., if (approximately)

$$
C=\frac{E_{0} S}{K_{m}+S}
$$

then thereafter the trajectory should closely follow this nullcline providing $t_{C} / t_{S} \ll 1$. During some time the order of magnitude of $S(t)$ is $S_{0}$. For this most important range of $S(t)$, condition (46a) becomes the parameter relation

$$
\frac{E_{0}}{K_{m}+S_{0}} \frac{1}{1+\kappa} \frac{1}{1+\left(S_{0} / K_{m}\right)} \ll 1, \quad \text { i.e., } \quad \epsilon \ll(1+\kappa)(1+\sigma) .
$$

(Note that (45c) is equivalent to (15) when $S=S_{0}$, while (46c) is equivalent to (16).) More generally the QSSA "nullcline following" is expected as long as $S(t)$ is such that $t_{C} / t_{S} \ll 1$, where $t_{C} / t_{S}$ is given in (46a).

We stress that conditions such as (46c) are consistency conditions for the QSSA, since the QSSA was employed in estimating $t_{S}$. Inconsistent approximations are generally invalid, while consistent approximations are generally valid unless the problem is ill-conditioned [30], [20, § 6.1].

4.4. The extended QSSA. As we have seen, a stricter condition than (46c), $\epsilon \ll 1$, results from the requirement that there is negligible diminution of the substrate concentration during the fast transient. Our reasoning implies that if

$$
\epsilon \approx 1
$$


but

$$
(1+\kappa)(1+\sigma) \gg 1
$$

then the QSSA

$$
\begin{aligned}
& c \approx \frac{(\sigma+1) s}{\sigma s+1}, \\
& \frac{d s}{d T} \approx-\frac{(\sigma+1) s}{\sigma s+1},
\end{aligned}
$$

would hold after the fast transient, as before. But in view of $(47 \mathrm{a})$ the initial condition $s(0)=1$ would not be appropriate for $(48 \mathrm{~b})$. This is exemplified by curves (c) and (d) in the numerical example of Fig. 1.

Note from Figs. 1 and 2 that the rapid decrease of $s$ and $c$ seems to occur along a straight line. This is a general phenomenon that allows us to formulate an extended QSSA. The reason is that if (47b) holds, then the right sides of (21a) and (21b) are nearly proportional to one another. Either $\kappa \gg 1$, in which case both right-hand sides are approximately proportional to

$$
-s+\frac{\sigma}{\sigma+1} c s+\frac{1}{\sigma+1} c
$$

or $\sigma \gg 1$ and the proportionality factor is $-s+c s$. Thus during the fast transient

$$
\frac{d c}{d s} \approx-\epsilon^{-1}
$$

giving the straight line

$$
c=\epsilon^{-1}(1-s)
$$

The intersection of (50) and (48a) determines the appropriate "initial" value for (48b):

$$
s(0)=s^{(i)}, \quad s^{(i)} \equiv \frac{\sigma-1-\epsilon(\sigma+1)+\left\{[\sigma-1-\epsilon(\sigma+1)]^{2}+4 \sigma\right\}^{1 / 2}}{2 \sigma} .
$$

The corresponding value of $c$, obtained from (48a) or (50), will be denoted by $c^{(m)}$, for it is an estimate of the maximum value of the dimensionless complex concentration:

$$
c^{(m)}=\epsilon^{-1}\left(1-s^{(i)}\right) .
$$

Table 2 shows simulation results that support the estimates (51) and (52) for $s^{(i)}$ and $c^{(m)}$. Table 2 only illustrates situations wherein $(47 \mathrm{~b})$ is attained for $\kappa \gg 1$, $\sigma=O(1)$. The alternative $\sigma \gg 1, \kappa=O(1)$ results in a small value of $s^{(i)}$ and hence a small and uninteresting domain of applicability of the extended QSSA.

Our main motivation here in presenting the extended QSSA is not to put forward a new approximation that we feel will have important practical applications. Rather we wish to stress the independence of the two requirements $t_{C} \ll t_{S}$ (assuming that the complex $C$ can change fast enough to keep pace with the changes in substrate $S$ ) and $\left|\Delta S / S_{0}\right| \ll 1$ (assuring that a negligible amount of substrate is consumed during the fast transient). 
4.5. The reverse QSSA. To further test our understanding of the principles we have been enunciating, we can ask, are there parameter domains where instead of $C$ being in a quasi-steady state with respect to $S$ there is a "reverse QSSA" in which $S$ is in a quasi-steady state with respect to $C$ ? Application of our scaling concepts shows that the answer is affirmative. See Appendix 3 for details.

5. Literature review. What amounted to the derivation of the basic formulae (4) and (5) was carried out by Henri [15] and Michaelis and Menten [21], nominally for the case when the product formation rate $k_{2}$ is sufficiently small, and by Briggs and Haldane [2] when the initial substrate concentration is sufficiently large compared to the initial complex concentration. We note that in fact the relative smallness of $k_{2}$ does not guarantee the validity of the QSSA, as was pointed out in [14].

Laidler [18] made an early attempt to determine conditions under which the QSSA is valid, including approximate solutions for the transient phase. In particular, he derived a bound

$$
\frac{P}{S_{0}}<\frac{5.3 k_{2}}{2 k_{1} S_{0}}\left[\frac{1}{(1-Q)^{1 / 2}}-1\right]
$$

for the amount of product produced during the transient phase, where

$$
Q \approx k_{1}^{2}\left[\left(E_{0}+S_{0}+K_{m}\right)^{2}-4 E_{0} S_{0}\right] .
$$

Surprisingly Laidler [18] deduced that $P / S_{0}$ is small, and hence the QSSA is valid, not only when

$$
S_{0} \gg E_{0} \quad \text { or } \quad K_{m} \gg E_{0}
$$

(in agreement with our results) but also when

$$
E_{0} \gg S_{0} \quad \text { or } \quad K_{m} \gg S_{0} .
$$

The incorrect conditions (55b) turn out to be consequences of Laidler's conclusion from (53) that $Q \ll 1$ is sufficient to yield $P \ll S_{0}$. Expansion of the square brackets in (53) yields the additional condition

$$
5.3 k_{2} Q \ll 4 k_{1} S_{0}
$$

Bowen, Acrivos, and Oppenheim [1] were the first to examine the QSSA with the aid of singular perturbation theory. After reviewing previous efforts to estimate the error in various quasi-steady-state assumptions, they analyzed four kinetic schemes, not including the enzyme-substrate-complex problem of (1). Dimensionless variables were introduced without motivation.

Wong [36] derived an expression for the relative error in making the central QSSA expression (4a), but in doing so he neglected terms of the same order as those retained. In essence, Wong [36] wrote

$$
C=\frac{E_{0} S}{K_{m}+S}(1+\Delta), \quad|\Delta| \ll 1 .
$$

He substituted this expression into (3b), obtaining for the two sides of the equation

$$
\begin{aligned}
& \frac{d C}{d t}=\frac{E_{0} K_{m}}{\left(K_{m}+S\right)^{2}} \frac{d S}{d t}(1+\Delta)+\frac{E_{0} S}{K_{m}+S} \frac{d \Delta}{d t}, \\
& k_{1}\left(E_{0}-C\right) S-\left(k_{-1}+k_{2}\right) C=-k_{1} E_{0} S \Delta .
\end{aligned}
$$


Upon neglecting terms proportional to $\Delta$ and $d \Delta / d t$ in (57b), Wong obtained an explicit approximate expression for the relative error $\Delta$ by equating $(57 \mathrm{~b})$ and $(57 \mathrm{c})$, and employing equation (5a) for $d S / d t$. But along with (57a) we must simultaneously consider a correction to $S$, and this provides an additional small term on the right side of (57c). The only consistent way to proceed is via series expansions, as in $\S 2$.

As we have implied, Heineken, Tsuchiya, and Aris [14] provided a careful discussion of the singular perturbation treatment of (1), giving references to earlier efforts in this direction. Seshadri and Fritzsch [33], unaware of [14], studied the more general situation wherein the reversible nature of the formation of product was taken into account. They employed dimensionless variables that differ from the standard set (7) in that $K_{m}$ is taken as the scale for the enzyme concentration. This yielded $E_{0} / K_{m}$ as the natural small parameter, which of course is a special case of our more general parameter $\epsilon$ of (22). As the source of their choice of dimensionless variables Seshadri and Fritzsch [33] cited Reich and Sel'kov [27], but the latter authors provided no motivation for this choice.

Klonowski [16] reviewed some of the Russian literature concerning approximations for chemical kinetics, describing theorems of Tikhinov and Korzuhin. There is a general discussion of timescales, but once again a virtually unmotivated use of the standard variables (7) is introduced in a treatment of the QSSA. Of great interest is the statement that the QSSA is valid either when $E_{0}$ is small compared to $S_{0}$ or when $k_{1}$ is small compared to $k_{-1}$ and $k_{2}$. This is very close to our result that $E_{0} /\left(K_{m}+S_{0}\right)$ is the appropriate small parameter of the problem. Unfortunately the present authors have not been able to inspect the two Russian books cited by Klonowski [16] as sources for his remarks concerning the validity of the QSSA.

Analog and digital computer simulations have been used by several researchers to study the validity of the QSSA. For example, Stayton and Fromm [35], who cite earlier work, simulated the reversible enzyme-substrate-complex equations and found that the QSSA is generally a good approximation when $E_{0} / S_{0}$ is less than 0.01 , while "normally" in experiments this ratio is two to four orders of magnitude smaller. However, Stayton and Fromm [35] pointed out that Sols and Marco [34] provide a list of instances illustrating the fact that in vivo $E_{0} / S_{0}$ is often of order unity. This testifies to the biological importance of our extension of the classical results to situations where $E_{0} /\left(K_{m}+S_{0}\right)$ is small. An additional example is found in the study of post-synaptic behavior by Parnas, Flashner, and Spira [26] where a system entirely analogous to (3) is studied.

Schauer and Heinrich [29] made a valuable study of errors in the QSSA. They proposed three criteria. These are the smallness of the relative relaxation deficit $\delta_{S} \equiv$ $\Delta S / S_{0}$, of the relative relaxation time $\delta_{T} \equiv t_{C} / t_{S}$, and of the relative relaxation error $\delta_{R}$, which we have defined in (31). Schauer and Heinrich [29] provided relatively accurate but correspondingly complex estimates of $\delta_{S}, \delta_{T}$, and $\delta_{R}$. Gratifyingly, these estimates correspond closely to our own much simpler formulae (Appendix 2).

The importance of scaling in numerical analysis, particularly in chemical kinetics, was stressed by Dahlquist and his associates [8], [37], [7]. In particular, Dahlquist et al. [8] pointed out that scaling "gives appropriate weights in the norm for the measuring of the local error (and) makes it possible to write the system in the partitioned form" (28), where $s$ and $c$ should be interpreted as vectors. Zu-fan [37] presented a systematic approach to scaling of equations with numerical coefficients whose right-hand sides are sums of products of powers of the variables. A small parameter is selected, and scales for each variable are defined in terms of unknown powers of this parameter. To fix the unknowns, in each equation a pairwise balance is sought (compare $\S 3$ ). Either 
the derivative term nearly balances one of the (algebraic) terms on the right side or there is a near balance between two algebraic terms of opposite sign; unbalanced terms will be relatively small. Determination of the unknown powers in order to make the balance as close as possible is a linear programming problem. Zu-fan [37] remarked that further information about the system must generally be added if this approach is to succeed. By contrast, we have seen that the Principle of Minimal Simplification is superior to the approach of pairwise balancing: it would be interesting to apply this Principle along the lines of [37].

Nipp [23], [24] presented a heuristic analytic algorithm for systematically obtaining approximate asymptotic solutions to a class of singular initial value problems. He based his approach on the observation that a change of scale can give meaningful limits as $\epsilon \rightarrow 0$ for exponents (of $\epsilon$ ) that lie on a certain convex polyhydral set that implies a similarity with the (later) approach of [37]. Nipp [24] eventually arrives at an algorithm containing 37 steps.

5.1. Center manifold. Carr [4] used the QSSA as an example in his treatment of center manifold theory. This theory produces useful reformulations of various problems in differential equations. The basic idea of the "global" theory is to cast the problem into the form

$$
\begin{aligned}
& \frac{d x}{d t}=A x+\epsilon f(x, y, \epsilon) \\
& \frac{d y}{d t}=B y+\epsilon g(x, y, \epsilon) \\
& \frac{d \epsilon}{d t}=0
\end{aligned}
$$

where $x \in R^{n}, y \in R^{m}$. Here $A$ and $B$ are constant matrices having eigenvalues with zero and negative real parts, respectively. If $f$ and $g$ are twice continuously differentiable with

$$
f(0,0,0)=0, \quad g(0,0,0)=0,
$$

then the relatively rapid expected decay of $y$ is manifested in the existence of an invariant manifold $y=h(x, \epsilon)$ for $|x|$ and $|\epsilon|$ sufficiently small, $h=O(\epsilon)$. The flow on the manifold is given by

$$
\frac{d u}{d t}=A u+f[u, h(u, \epsilon), \epsilon]
$$

where $h$ can be explicitly approximated to arbitrary accuiacy.

In his analysis of the QSSA via center manifold theory, Carr [4] started with the dimensionless equations of [14]. It is instructive to apply Carr's treatment to our dimensionless equations (21). In these variables, the QSSA assumption $d c / d \tau \approx 0$ yields

$$
s-\frac{c}{\sigma+1}(\sigma s+1)=0
$$

We thus introduce a variable $v$ that measures the deviation from the quasi-steady state:

$$
v=s-\frac{c}{\sigma+1}(\sigma s+1)
$$


With this, (21b) yields $d c / d \tau=v$ so that

$$
\frac{\sigma+1}{\sigma s+1} \frac{d v}{d \tau}=\frac{(\sigma+1)(\sigma v+1)}{(\sigma s+1)^{2}} \frac{d s}{d \tau}-v
$$

Since $d s / d \tau=O(\epsilon)$ by (21a), to obtain an equation for $v$ having the desired form (58b) we introduce a new time variable $\theta$ by

$$
\frac{d \tau}{d \theta}=\frac{\sigma+1}{\sigma s+1}
$$

This results in equations of the appropriate structure

$$
\begin{aligned}
& \frac{d v}{d \theta}=-v+\epsilon(\sigma+1)(1+\sigma v) \frac{\left[\kappa(\kappa+1)^{-1}-\sigma v-1\right] s-\kappa(\kappa+1)^{-1} v}{(\sigma s+1)^{3}} \\
& \frac{d s}{d \theta}=\epsilon(\sigma+1) \frac{\left[\kappa(\kappa+1)^{-1}-\sigma v-1\right] s-\kappa(\kappa+1)^{-1} v}{(\sigma s+1)^{2}} \\
& \frac{d \epsilon}{d \theta}=0 .
\end{aligned}
$$

With the equations in the form (65), it is particularly transparent that $v$ should rapidly decrease, yielding a center manifold with structure

$$
v=\epsilon f(s)+O\left(\epsilon^{2}\right) \text {. }
$$

Upon substitution of (66a) into (65a) we obtain

$$
v=-\epsilon(\sigma+1)(\kappa+1)^{-1} s(1+\sigma s)^{-3}+O\left(\epsilon^{2}\right) .
$$

Flow on the center manifold is found by substituting (66b) into (65b). This gives to lowest order

$$
\frac{d s}{d \theta}=-\frac{\epsilon(\sigma+1)(\kappa+1)^{-1} s}{(\sigma s+1)^{2}}
$$

or

$$
\frac{d s}{d \tau}=-\frac{\epsilon}{1+\kappa} \frac{s}{\sigma s+1}
$$

If definitions (20c) and (23) for $\tau$ and $T$ are employed, it is at once seen from (42) that $(67 \mathrm{~b})$ is equivalent to the Michaelis-Menten equation (28b).

Starting from the form (66), Carr [4] proceeded to provide a proof that the QSSA is valid. But this proof does not deal with the case of biological interest, for it treats situations where the substrate concentration has substantially decayed from its initial value. In the next section we provide a proof that does relate to the appropriate range of substrate concentrations.

6. Convergence proof. In this section we prove a theorem that describes the behavior of $s$ and $c$ on both the fast and slow timescales. 
THEOREM. Assume that $\sigma$ and $\kappa$ are restricted to the parameter ranges

$$
\begin{aligned}
& 0 \leq \sigma \leq \sigma_{0} \\
& 0 \leq \kappa \leq \kappa_{0} \\
& 0<q_{0} \leq \sigma+\kappa(\kappa+1)^{-1}
\end{aligned}
$$

for positive constants $\sigma_{0}, \kappa_{0}, q_{0}$. Then the solutions of (21) satisfy

$$
\begin{aligned}
& \left|\frac{d s}{d \tau}-\epsilon \frac{\left[\kappa(\kappa+1)^{-1}-1\right]}{(\sigma s+1)} s\right| \leq \text { const. }\left[\epsilon^{2}+\epsilon \exp \left(-\tau\left(\frac{1-\delta_{0}}{1+\sigma_{0}}\right)\right)\right], \\
& \left|s-\frac{c}{(\sigma+1)}(\sigma s+1)\right| \leq \text { const. }\left[\epsilon+\exp \left(-\tau\left(\frac{1-\delta_{0}}{1+\sigma_{0}}\right)\right)\right],
\end{aligned}
$$

for all $\tau \geq 0$. Furthermore, solutions of (24) satisfy

$$
\begin{aligned}
& \left|\frac{d s}{d T}+\frac{(\sigma+1) s}{\sigma s+1}\right| \leq \text { const. }\left[\epsilon+\exp \left(\left(\frac{1-\delta_{0}}{1+\sigma_{0}}\right)\left(\frac{-T}{\epsilon}\right)\right)\right], \\
& \left|s-\frac{c}{\sigma+1}(\sigma s+1)\right| \leq \text { const. }\left[\epsilon+\exp \left(\left(\frac{1-\delta_{0}}{1+\sigma_{0}}\right)\left(\frac{-T}{\epsilon}\right)\right)\right] .
\end{aligned}
$$

In addition, as $\epsilon \downarrow 0$, on any interval $0<T_{0} \leq T \leq T_{1}<\infty$ solutions of (24) converge uniformly in $\sigma, \kappa, T$ to a solution of the Michaelis-Menten equation (28b), $s_{0}(T)$, and to a function $c_{0}(T)$, where $c_{0}$ and $s_{0}$ satisfy (28a), i.e.,

$$
c_{0}=\frac{(\sigma+1) s_{0}}{\sigma s_{0}+1} .
$$

In the above "const." denotes some constant depending at most on $\sigma_{0}, \kappa_{0}, q_{0}$, while $\delta_{0}$ is a constant satisfying $0 \leq \delta_{0}<1$.

Proof. Introduce the variables $v, \theta$ as in (62), (64) with the normalization $\tau(0)=$ 0 . Then $v, s$ satisfy $(65 \mathrm{a}),(65 \mathrm{~b})$ and $v(0)=1, s(0)=1$. Also from (65b) we see that

$$
\frac{d s}{d \theta} \lesseqgtr 0
$$

when

$$
v \gtreqless \frac{(\delta-1) s}{\delta+\sigma s} .
$$

Here we have adopted the abbreviation $\delta=\kappa(\kappa+1)^{-1}$. Furthermore, since (65a) has the form

$$
\frac{d v}{d \theta}=-v+\frac{\epsilon(\sigma+1)(1+\sigma v)}{(\sigma s+1)} \cdot \frac{d s}{d \theta}
$$

we see that if $v>-\sigma^{-1}, s>-\sigma^{-1}$ then

$$
\frac{d v}{d \theta} \lessgtr 0
$$

when

$$
v \gtrless 0 \quad \text { and } \quad v \gtreqless \frac{(\delta-1) s}{\delta+\sigma s} \text {. }
$$


From $(70),(71)$ we see that the evolution of $(65 \mathrm{a}),(65 \mathrm{~b})$ may be illustrated by the phase-plane portrait shown in Fig. 3.

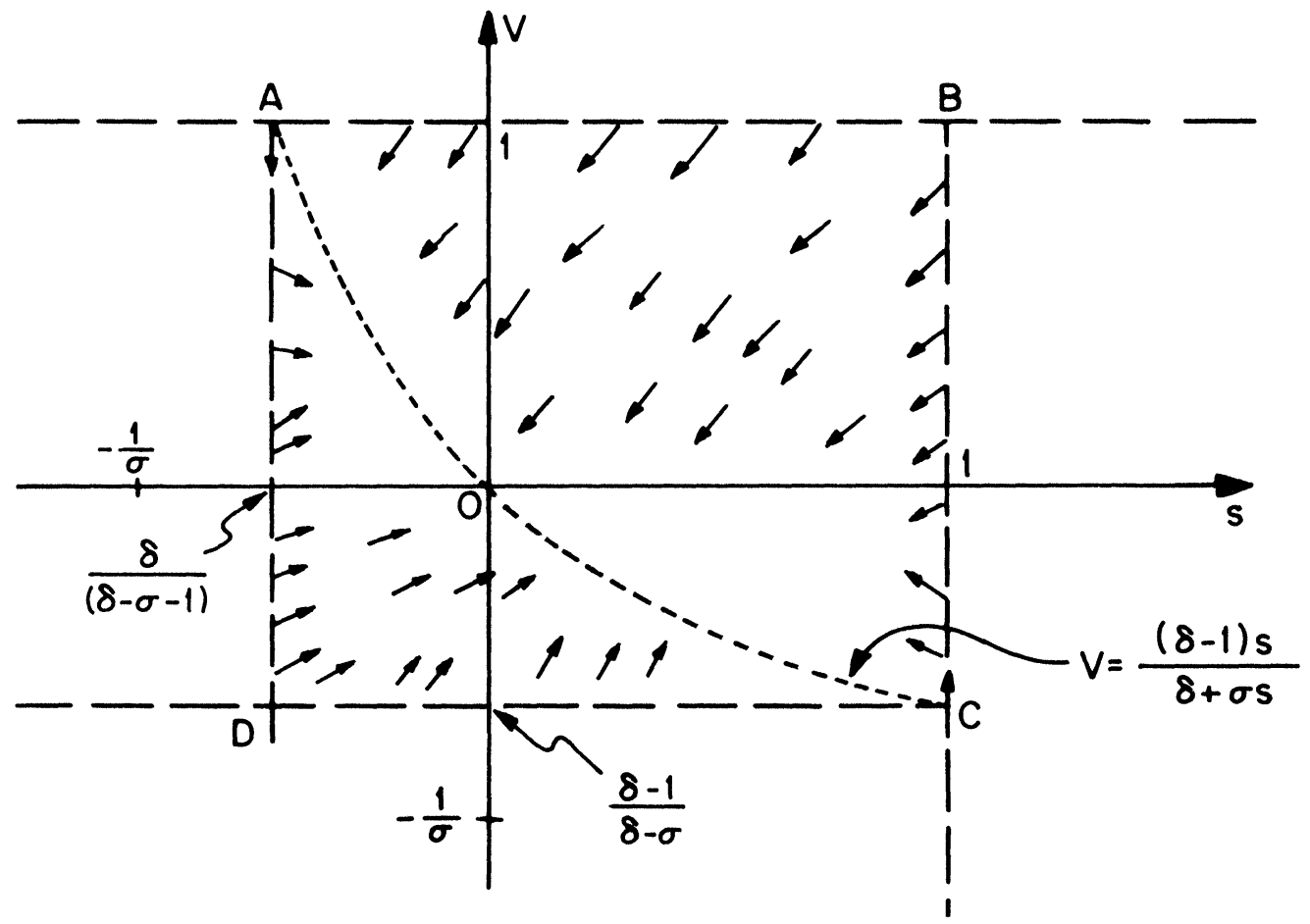

FIG. 3. Sketch of phase plane for (65a), (65b). The dashed curve is the vertical nullcline on which $d s / d \theta=0$. The closed rectangle $\mathrm{ABCD}$ is positively invariant.

In particular (70), (71) show that the closed rectangle $\mathscr{R}$ whose vertices are denoted ABCD is positively invariant, i.e., any solution of $(65 \mathrm{a}),(65 \mathrm{~b})$ with initial data in $\mathscr{R}$ at $\theta=0$ will remain in $\mathscr{R}$ for all $\theta>0$. Also note that the ordering on the axes shown in Fig. 3 is indeed correct, i.e.,

$$
\begin{aligned}
& \frac{\delta}{\delta-\sigma-1}>-\frac{1}{\sigma} \\
& \frac{\delta-1}{\delta+\sigma}>-\frac{1}{\sigma} .
\end{aligned}
$$

This is because $(72 \mathrm{a})$ is equivalent to $(1-\delta)(1+\sigma)>0$, which is true because $0 \leq \kappa \leq \kappa_{0}$ and $\sigma \geq 0$, and (72b) is equivalent to $\delta \sigma>-\delta$.

From the positive invariance of $\mathscr{R}$ we know that if $s(0), v(0)$ are in $\mathscr{R}$ then for all $\theta>0$

$$
\begin{aligned}
& -\frac{(1-\delta)}{\delta+\sigma} \leq v \leq 1 \\
& -\frac{\delta}{1-\delta+\sigma} \leq s \leq 1
\end{aligned}
$$

Since $0 \leq \kappa \leq \kappa_{0}<\infty$ there is a positive constant $\delta_{0}$ such that $0 \leq \delta \leq \delta_{0}<1$. Hence (73) implies that for all $\theta>0$

$$
-\frac{1}{q_{0}} \leq v \leq 1
$$




$$
-\frac{\delta_{0}}{1-\delta_{0}} \leq s \leq 1
$$

Furthermore note that (73b) implies

$$
\sigma s+1 \geq \frac{-\sigma \delta}{1-\delta+\sigma}+1=(1-\delta)\left(\frac{\sigma+1}{1-\delta+\sigma}\right) \geq 1-\delta \geq 1-\delta_{0}
$$

for all $\theta>0$.

If we again denote by "const." any constant depending on at most $\sigma_{0}, \kappa_{0}, q_{0}$, we now see that (65a), (65b), (73), (74) and (75) imply that for $\theta>0, v, s$ satisfy

$$
\begin{aligned}
& \frac{d v}{d \theta}=-v+\epsilon F(v, s), \\
& \frac{d s}{d \theta}=\epsilon G(v, s),
\end{aligned}
$$

where $|F(v, s)| \leq$ const., $|G(v, s)| \leq$ const. From (76a) we see that

$$
\left|v(\theta)-e^{-\theta}\right| \leq \epsilon \text { const. }
$$

Substitution of (77) into (65b) shows that

$$
\left|\frac{d s}{d \theta}-\epsilon(\sigma+1) \frac{\left[\kappa(\kappa+1)^{-1}-\sigma e^{-\theta}-1\right] s-\kappa(\kappa+1)^{-1} e^{-\theta}}{(\sigma s+1)^{2}}\right| \leq \epsilon^{2} \text { const. }
$$

But since

$$
\frac{d \theta}{d \tau}=\frac{\sigma s+1}{\sigma+1}, \quad \theta(0)=0
$$

we know that

$$
\frac{1-\delta_{0}}{\sigma_{0}+1} \leq \frac{d \theta}{d \tau} \leq 1
$$

and hence

$$
\tau\left(\frac{1-\delta_{0}}{1+\sigma_{0}}\right) \leq \theta \leq \tau, \quad \exp (-\tau) \leq \exp (-\theta) \leq \exp \left[-\tau\left(\frac{1-\delta_{0}}{1+\sigma_{0}}\right)\right] .
$$

Equations (69a) and (69b) now follow, proving the first part of the theorem.

Next substitute $T=\epsilon \tau /(\kappa+1)(\sigma+1)$ so that $(69 \mathrm{a})$ and $(69 \mathrm{~b})$ imply

(80a) $\left|\frac{d s}{d T}+\frac{(\sigma+1) s}{\sigma s+1}\right| \leq$ const. $\left[\epsilon+\exp \left(\frac{(\kappa+1)(\sigma+1)\left(1-\delta_{0}\right)}{\left(1+\sigma_{0}\right)}\left(-\frac{T}{\epsilon}\right)\right)\right]$
(80b) $\left|s-\frac{c}{(\sigma+1)}(\sigma s+1)\right| \leq$ const. $\left[\epsilon+\exp \frac{(\kappa+1)(\sigma+1)\left(1-\delta_{0}\right)}{\left(1+\sigma_{0}\right)}\left(-\frac{T}{\epsilon}\right)\right]$.

Since $\kappa \geq 0, \sigma \geq 0$ the above inequalities imply (69c) and (69d), proving the second part of the theorem.

Finally, on any interval $0<T_{0} \leq T \leq T_{1}<\infty$ let $\left\{s_{\epsilon}(T), c_{\epsilon}(T)\right\}$ denote the sequence of solutions of (24), parametrized by $\epsilon>0$. Inequality (69c) shows (after a possible trivial redefinition of $\epsilon$ ) that $s_{\epsilon}(T)$ is an $\epsilon$-approximate solution, in the sense of the polygonal method for construction of solutions, to the Michaelis--Menten equation 
(28b). (See [6], for example). Hence the standard argument of the proof of the CauchyPeano existence theorem shows the sequence $\left\{s_{\epsilon}(T) ; \epsilon>0\right\}$ has a subsequence that converges uniformly in $\sigma, \kappa, T$ to a solution $s_{0}(T)$ of (28b). Furthermore, the only way the original sequence cannot itself converge to $s_{0}(T)$ is if there is a second subsequence of $\left\{s_{\epsilon}(T) ; \epsilon>0\right\}$ that converges to a second distinct solution of $(28 \mathrm{~b})$. But inspection of $(28 \mathrm{~b})$ shows that all solutions starting at $T_{1}$ with data $s\left(T_{1}\right)$ must lie in the interval $0 \leq s(T) \leq s\left(T_{1}\right)+1$. On this interval the right-hand side of (28b) is continuously differentiable, hence Lipschitz continuous in $s$. So the standard uniqueness theorem of ordinary differential equations tells us that (28b) possesses unique solutions, so that $\left\{s_{\epsilon}(T)\right\}$ converges to $s_{0}(T)$ uniformly in $\sigma, \kappa, T$. This completes the proof of the theorem.

7. Summary and discussion. We believe that our analysis has resulted in a considerably clearer understanding of an important particular matter, the classical quasi-steady state assumption (QSSA) of biochemical kinetics, and also that our discussion points the way to a small improvement in a general technique in applied mathematics.

As we have indicated, a number of authors have been concerned with numerical error estimates for the QSSA. Quite accurate results have been obtained, but here we present estimates that are both satisfactorily accurate and also very simple. Since other error measures either are smaller or are appropriate only after the substrate has markedly decreased, the decisive error measure turns out to be $\delta_{S}$, the fractional decrease in substrate during the pre-steady-state transient. It follows as a rule of thumb that if a fractional error $\delta_{S}$ can be tolerated (e.g., for a 10 percent error $\left.\delta_{S}=0.1\right)$ then the QSSA can be employed if

$$
\epsilon \equiv \frac{E_{0}}{K_{m}+S_{0}}<\delta_{S}
$$

This error bound is not rigorous, but we have presented evidence that it is generally rather accurate. We remark that a numerical error estimate is particularly helpful to experimentalists, and it is rare that one can be obtained. C.f. the famous "dialogue" in $[9$, p. 19].

We have presented a convergence theorem that is an improvement on earlier results in two ways: a more "liberal" small parameter is employed $\left[E_{0} /\left(K_{m}+S_{0}\right)\right.$ rather than $E_{0} / S_{0}$ ], and the results are uniform for a certain domain of the other two dimensionless parameters that characterize the problem.

Are the uniformity results the best that can be expected? One test is to check the "worst cases." In the uniformity condition (68) both nonnegative parameters $\sigma$ and $\kappa$ must be bounded from above. The "worst" conditions $\sigma=\infty$ and $\kappa=\infty$ occur if

$$
K_{m}=0, \quad \text { i.e., } k_{-1}+k_{2}=0 \quad \text { or } k_{2}=0 .
$$

Both equations of (82) imply $k_{2}=0$. In this case the QSSA (5) reduces to $S \equiv S_{0}$, certainly an inadequate approximation to the substrate-complex interchange. Condition (68c) rules out the simultaneous vanishing of $\sigma$ and $\kappa$. But $\sigma=0$ if and only if $S_{0}=0$, and the approximations are vacuous in this trivial case.

As the "worst cases" are indeed pathological, we conclude that no better uniformity results are to be expected. This conclusion is partially reinforced by the formal error analysis at the end of $\S 2$, which indicates that the absolute error can be significant if $\sigma=O\left(\epsilon^{-1}\right)$. 
Let us examine a little more closely the connection between the "standard" approach of [14] and ours. The relations between the standard parameters $\epsilon_{h}, \mu$, and $\lambda$ of (9) and the recommended parameters $\epsilon, \kappa$, and $\sigma$ of (22) and (17) are

$$
\begin{aligned}
& \epsilon_{h}=\epsilon\left(1+\sigma^{-1}\right), \\
& \mu=\sigma^{-1} \\
& \lambda=[\sigma(1+\kappa)]^{-1},
\end{aligned}
$$

and

$$
\begin{aligned}
& \epsilon=\epsilon_{h}(1+\mu)^{-1} \\
& \sigma=\mu^{-1} \\
& \kappa=(\mu / \lambda)-1
\end{aligned}
$$

The standard dependent variables $y, z$, and inner and outer time variables $H$ and $h$ of (7) and (10) are related to their counterparts of (20) and (23) by

$$
y=s, \quad z=\sigma(\sigma+1)^{-1} c, \quad H=\sigma(\sigma+1)^{-1} \tau, \quad h=(\sigma+1)(\kappa+1) T
$$

The small parameters $\epsilon_{h}$ and $\epsilon$ enter the above equations only in the mutual relations (83a) and (84a). Thus there is no reordering of terms in the various power series expansions upon passing back and forth between the standard and the recommended variables. Thus we are not concerned here with a situation wherein a partial summation of terms promotes better convergence. Rather, with correctly scaled variables we can recognize the true expansion parameter whose smallness guarantees convergence, since (by definition of "correctly scaled") the dependent variables will be $O(1)$.

We obtained our results via estimates of the scales of the variables, i.e., estimates of the fast and slow timescales and of the scales of the dependent variables. With these scales, suitable dimensionless variables could be defined. The scaling estimates employed a certain grasp of the nature of the solution, but it cannot be said that a profound understanding was required. It is thus remarkable that in essence no motivation was advanced in any of the previous introductions of dimensionless variables as part of arguments leading up to the QSSA.

There is an "exception that proves the rule" with respect to motivation in introducing dimensionless variables. One of the authors attempted such a motivation (see [20, pp. 306-307] but the results were partial and unconvincing. The "obvious" choice of timescales became manifest later [31] and the level of understanding reported here only arose after further thought.

It appears that there is a lesson to be drawn from the history of the QSSA. Earlier authors apparently did not make a major effort to arrive at appropriately scaled variables. Now an effort has been made, but it only succeeded after some time. The moral seems to be that scaling is not as easy as it might appear, and thus 
the applied mathematical community should strive to improve understanding of this partially intuitive technique. It would be helpful if all of us tried to make as explicit as possible the reasoning that leads us to a choice of scaled dimensionless variables.

The present problem exemplifies the extremely common situation where a first approach reveals the possibility of exploiting, in some sort of perturbation procedure, the fact that one of the several dimensionless parameters of the problem is small. In such cases we assert that the analyst should not rest, assuming that the problem is important, until variables are selected in terms of which the perturbation procedure seems valid (or has been proved to be valid), to the maximal extent, uniformly in the other parameters.

The methods employed here can be applied to a number of similar situations, such as the enzyme-substrate-inhibitor system (see, [28, § 2.3] and [32]). Frenzen and Maini [12] have applied these methods to a two-step enzymatic reaction when $E_{0} \approx S_{0}$ but $\epsilon \ll 1$.

We conclude with two generalizations about scaling that can be gleaned from the present study. These generalizations can be added to the essential points concerning scaling that were listed at the end of the Introduction.

Supplement to point (ii). When there are several dependent variables with different modes of variation, choose the scale of the independent variables with respect to the fastest varying dependent variable. Then (as in (21)) the scaled equations will automatically reflect the relative slowness of changes in the other dependent variables.

Additional point (viii). (This is to enable the goal, mentioned three paragraphs earlier, of obtaining approximations that are uniformly valid in the "nonsmall" parameters.) Suppose, to take the simplest example, that the parameter domain for which the scalings are valid (see $(\mathrm{v})$ ) can be written $0<\epsilon \ll 1$. Then the estimates embodied in the scales ideally should be accurate for small $\epsilon$ up to a factor that is of order unity uniformly in the other parameters of the problem.

Appendix 1. More accurate estimation of $\left|\Delta S / S_{0}\right|$. A more accurate estimate than $(18 \mathrm{a})$ is

$$
\left|\frac{\Delta S}{S_{0}}\right| \approx \frac{1}{S_{0}} \cdot \frac{1}{2}\left[\left|\frac{d S}{d t}\right|_{\max }+\left|\frac{d S}{d t}\right|_{\min }\right] t_{C}
$$

where the max and min are taken over the pre-steady-state interval. Employing (5a) with $S=S_{0}$ to estimate the minimum value of $|d S / d t|$ in this interval we find

$$
\left|\frac{\Delta S}{S_{0}}\right|=\frac{1}{2}\left[\frac{E_{0}}{K_{m}+S_{0}}+\frac{k_{2} E_{0}}{k_{1}\left(K_{m}+S_{0}\right)^{2}}\right]=\frac{1}{2} \frac{\eta}{1+\sigma}\left[1+\frac{1}{(1+\kappa)(1+\sigma)}\right] .
$$

Thus (particularly since the factor $\frac{1}{2}$ is immaterial in our order of magnitude estimates) condition (19) indeed assures that $\left|\Delta S / S_{0}\right| \ll 1$.

Appendix 2: Error estimates. We shall compare our estimates of $\delta_{S}, \delta_{T}$, and $\delta_{R}$ with those of Schauer and Heinrich [29]. For example, they found after some calculation that

$$
t_{S} \approx \frac{9 K_{m} S_{1}+12 K_{m}^{2}+2 S_{1}^{2}}{6 k_{2} E_{0}\left(S_{1}+2 K_{m}\right)}, \quad S_{1} \equiv S_{0}-\Delta S .
$$

Since $S_{1} \approx S_{0}$ this estimate can be written as 


$$
t_{S} \approx \frac{1}{k_{2} E_{0}}\left[\frac{S_{0}}{3}+\frac{K_{m}}{6}\left(5+\frac{2}{2+S_{0} / K_{m}}\right)\right]
$$

We have sought simple order of magnitude estimates and have neglected $O(1)$ factors. Thus our estimate (15) is in line with (A2.1).

Let us pursue this matter a little further. The ratio $R$ of the Schauer-Heinrich estimate (A2.1) to our estimate (15) is

$$
R \equiv\left[2 \sigma+5+\left(1+\frac{1}{2} \sigma\right)^{-1}\right] /(6+6 \sigma)
$$

This quantity is of order unity for all nonnegative $\sigma$ : in particular $R=1$ when $\sigma=0$ and $R \rightarrow \frac{1}{3}$ as $\sigma \rightarrow \infty$. Had we used the Schauer-Heinrich estimate (A2.1) for $t_{S}$ in (23) instead of our estimate (15), then the equations would have been more complicated but (after obvious rearrangements) the right sides would have remained of order unity as functions of $\sigma$ for $0 \leq \sigma \leq \infty$. At the end of $\S 6$ we pointed out that the appropriate singular perturbation approximation to the solutions of (24) should be valid (as $\epsilon \rightarrow 0$ ) uniformly in $\sigma$ for $0 \leq \sigma \leq \sigma_{1}<\infty$. This expectation would not be altered by adoption of a new "more accurate" timescale based on (A2.1). This illustrates the point that in choosing scaled variables relevant to $0<\epsilon \ll 1$ for some parameter $\epsilon$ it is sufficient to strive for estimates that are accurate to within a factor that is of order unity uniformly in the other parameters of the problem.

For the fast timescale, Schauer and Heinrich [29] derive the approximate formula

$$
t_{C} \approx-\frac{1}{k_{1} W \ln (b+1)} \sum_{n=1}^{\infty} \frac{(-b)^{n}}{n^{2}}
$$

where

$$
\begin{aligned}
& W=\left[\left(K_{m}+E_{0}+S_{0}\right)^{2}-4 E_{0} S_{0}\right]^{1 / 2}, \\
& b=\frac{W-K_{m}-E_{0}-S_{0}}{W+K_{m}+E_{0}+S_{0}} .
\end{aligned}
$$

We expect that $b$ will be small. (For small $\epsilon, b$ decreases from less than 0.2 to zero as $\sigma$ increases from zero to $\infty$.) Thus, an approximation to (A2.3) is

$$
t_{C} \approx \frac{b}{k_{1} W}=\frac{b}{k_{1}\left(S_{0}+K_{m}\right)}\left[1+\frac{1}{(1+\sigma)^{2}}-\frac{2 \epsilon \sigma}{1+\sigma}+\epsilon^{2}\right]^{1 / 2} .
$$

It follows that, paralleling the case of $t_{S}$, our simple formula (13) is of the same magnitude as Schauer and Heinrich's much more complicated estimate (A2.3).

With (A2.1) and (A2.3) we can compute Schauer and Heinrich's estimate for $\delta_{T}$. Computer iterations are required to compute their estimates for $\delta_{S}$ and $\delta_{R}$. In Fig. 4 we exhibit their numerical results for the particular parameter values that they consider. (Note that if $(\log \sigma, \log \eta)$ falls below a given curve then the corresponding error is less than one percent.) These graphs should be compared with our results from (18b), (13) and (15), and (18): 


$$
\begin{aligned}
\delta_{S} & =\frac{f_{S} E_{0}}{K_{m}+S_{0}}, \\
\delta_{T} & =\frac{f_{T} k_{2} E_{0}}{k\left(K_{m}+S_{0}\right)} ; \\
\delta_{R} & =\max _{S} \frac{f_{R} k_{2} E_{0}}{k_{1}} \frac{K_{m} S}{\left(K_{m}+S\right)^{4}} \approx \frac{\left(0.1 E_{0} / K_{m}\right) f_{R}}{1+\left(k_{-1} / k_{2}\right)} .
\end{aligned}
$$

Here $f_{S}, f_{T}$, and $f_{R}$ represent $O(1)$ factors that were omitted in our estimates.

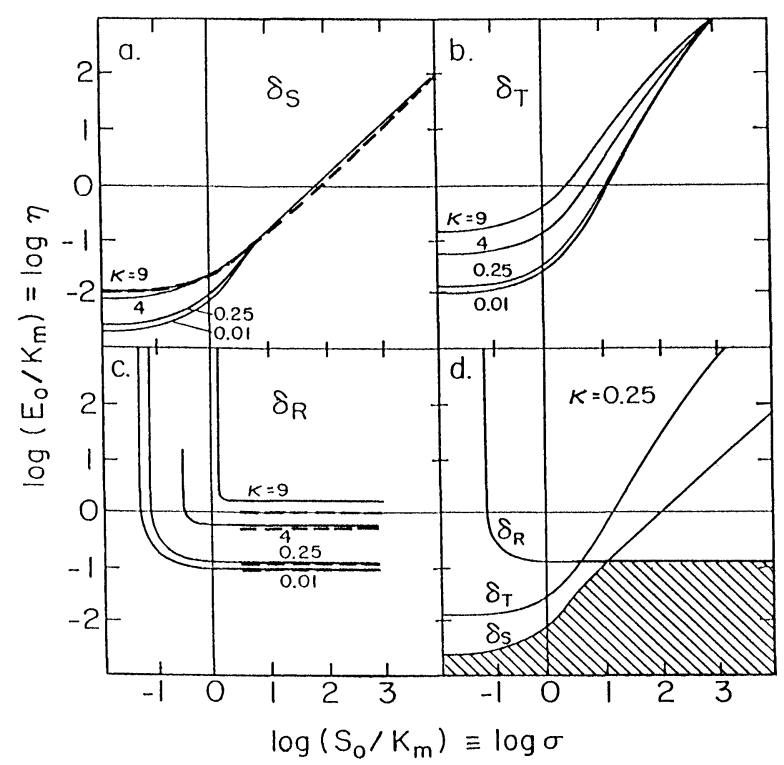

FIG. 4. Lines of constant 1 percent errors for different values of $\kappa=k_{-1} / k_{2}$ when $K_{m}=1 \mathrm{mM}$, $k_{1}=1 \mathrm{mM}^{-1} \mathrm{sec}^{-1}$. Solid lines taken from [29]. Log-log plots in the plane of $\eta \equiv E_{0} / K_{m}$ and $\sigma \equiv S_{0} / K_{m}$. (a) Relative initial transient decrease in substrate: $\delta_{S} \equiv \Delta S / S_{0}=0.01$. Dashed line: the simple estimate (A2.9), which is equivalent to (18b). (b) Ratio of transient duration to duration of QSSA: $\delta_{T}=t_{C} / t_{S}=0.01$. (c) Largest relative error in the reaction velocity $d P / d t$ (normalized to the maximum velocity $\left.k_{2} E_{0}\right): \delta_{R}=0.01$. Dashed lines: from our simple estimate (A2.6) with $f_{R}=1$. (d) Graphs of $\delta_{R}=\delta_{T}=\delta_{S}=0.01$ for $\kappa=0.25$. The QSSA is valid to within 1 percent when parameters fall in the shaded region.

Comparison of Figs. 4(a) and 4(b) confirms our earlier conclusion that if $\delta_{S}$ is small then $\delta_{T}$ is small, so that we shall not consider $\delta_{T}$ further. As to our estimate of $\delta_{S}$, if we make the definitions

$$
y \equiv \log \left(\frac{E_{0}}{K_{m}}\right), \quad x \equiv \log \left(\frac{S_{0}}{K_{m}}\right),
$$

(where logs are base 10) then (A2.6) yields

$$
y=\log \left(1+10^{x}\right)+\log \delta_{S}-\log f_{S} .
$$


In Fig. $4(\mathrm{a}), \delta_{S}=0.01$, and $y=0$ when $x=2$. Thus we may take $f_{S}=1$. Hence (since $y(-2)=-2$ )

$$
y=\log \left(1+10^{x}\right)-2
$$

well approximates the numerical results of Fig. 4(a). Thus since the numerical calculations show that the correction factor $f_{S}$ can in fact be taken to be unity we now expect that our basic estimate (18b) will provide rather good quantitative accuracy. Our more accurate estimate (A1.1) well reflects the trend of Fig. 4(a) for $\delta_{S}$ to decrease with $\kappa$ when $S_{0}$ is small.

Comparison of our estimate (A2.6) with Fig. 4 shows once again that even if the correction factor $f_{R}$ is taken to be unity there is very good agreement with the numerical results. The comparison is made only for large $S_{0}$, since with $f_{S}=f_{R}=1$, (A2.6) implies that $\delta_{R}=\delta$ ( $\delta$ a constant) lies beneath $\delta_{S}=\delta$ if

$$
\frac{S_{0}}{K_{m}} \equiv \sigma \geq 9+10 \kappa
$$

Note the good agreement of this result with Fig. 4(d).

Appendix 3: Aspects of the reverse QSSA. We seek conditions for which the substrate $S$ is, after a transient, in a quasi-steady-state with the complex $C$. From (3a) with $C$ fixed, we note that the intrinsic $S$ timescale with respect to $C$ is (employing a tilde to distinguish this example)

$$
\tilde{t}_{S}=\frac{1}{k_{1}\left(E_{0}-C\right)}
$$

The reverse QSSA is based on the assumption $d S / d t=0$ in (3a), yielding

$$
S=\frac{k_{-1}}{k_{1}} \frac{C}{E_{0}-C}
$$

On substitution of this expression for $S$ into (3b) we find

$$
\frac{d C}{d t}=-k_{2} C
$$

Thus the timescale for changes in $C$, assuming the QSSA holds, is

$$
\tilde{t}_{C}=k_{2}^{-1}
$$

We expect that (A3.2) will be valid after a relatively fast transient, if

$$
\tilde{t}_{S} \ll \tilde{t}_{C}
$$

that is if

$$
E_{0}-C \gg \frac{k_{2}}{k_{1}}
$$


Decrease in the complex concentration will ultimately yield condition (A3.5b) if

$$
E_{0} \gg \frac{k_{2}}{k_{1}}
$$

Note that if the reverse QSSA equation (A3.2) is solved for $C$, we obtain the familiar saturating form

$$
C=\frac{E_{0} S}{(K+S)},
$$

$$
\text { where } K \equiv \frac{k_{-1}}{k_{1}} \text {. }
$$

Equation (A3.7a) is indistinguishable from the corresponding QSSA equation (4a) if $k_{2} \ll k_{-1}$. Hence we restrict further consideration to cases wherein
(A3.8a)
$k_{-1} \approx k_{2}$
(A3.8b)
i.e., $\kappa \approx 1$.

Combining (A3.8) and (A3.6), we thus conclude that "interesting" cases of the reverse QSSA occur when

$$
E_{0} \gg K_{m}
$$

Figure 5 shows an example of a reverse QSSA. Note that both the fast transient and the later slow decay proceed along straight lines in the $C-S$ phase plane. Since $E_{0}$ is relatively large, we indeed expect the fast transient to be governed by

$$
\frac{d S}{d t}=-k_{1} E_{0} S, \quad \frac{d C}{d t}=k_{1} E_{0} S .
$$

This gives

$$
\begin{aligned}
& \frac{d C}{d S}=-1, \\
& C=S_{0}-S .
\end{aligned}
$$

The "knee" where rapid traverse of (A3.10) gives way to slow traverse of (A3.7a) will occur when

$$
S_{0}-S=\frac{E_{0} S}{(K+S)}
$$




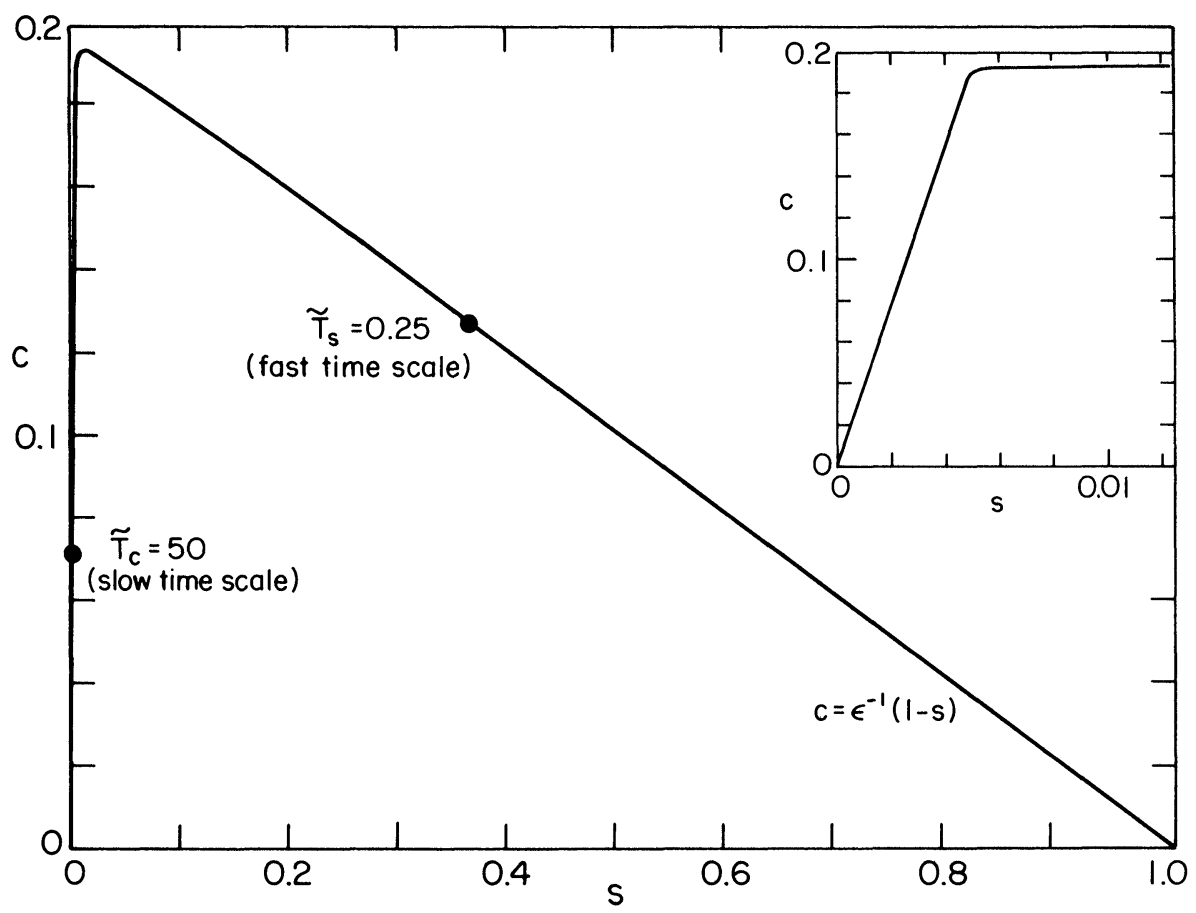

FIG. 5. Illustration of a reverse QSSA. Solution of the dimensionless equations (24) with $\epsilon=50, \sigma=1, \kappa=1$. Inset shows detail of the "post-knee" reverse QSSA. In the variables of (24), (A3.13), (A3.10b), (A3.12a), (A3.12b) take the form $C=(\sigma+1)\left(1+\kappa^{-1}\right) S, c=\epsilon^{-1}(1-s)$, $s_{k}=\left[\epsilon(1+\sigma)\left(1+\kappa^{-1}\right)\right]^{-1}, c_{k}=\epsilon^{-1}$, with fast and slow timescales $\tilde{T}_{S}=[(1+\sigma)(1+\kappa)]^{-1}$, $\tilde{T}_{C}=\epsilon$. With present parameter values we have to first approximation the reverse QSSA $c=4 s$, the transient $c=0.02(1-s)$, the predicted "knee" coordinates $s_{k}=0.005$ and $c_{k}=0.02$, and the timescales $\tilde{T}_{S}=0.25$ and $\tilde{T}_{C}=50$.

For definiteness we consider the special case (as in Fig. 5) wherein $S_{0} \approx K_{m}$ so that, in addition to (A3.9), $E_{0} \gg S_{0}$. (Thus $\epsilon \equiv E_{0} /\left(S_{0}+K_{m}\right) \gg 1$.) Then, on solving (A3.11) we find that the coordinates of the "knee" are approximately

$$
\begin{aligned}
S_{k} & \approx \frac{S_{0} K}{E_{0}}, \\
C_{k} & \approx S_{0} .
\end{aligned}
$$

Since $S_{k} \ll S_{0}$, the reverse QSSA equation (A3.7a) can be simplified to

$$
C=\frac{E_{0}}{K} S
$$

which indeed is a straight line. From (A3.a), (A3.12b) and the assumption $E+0 \gg$ $S_{0}$ it follows from condition (A3.5b) that the reverse QSSA (A3.13) should be valid throughout the entire post-transient period. As shown in Fig. 5, the various qualitative and quantitative predictions are borne out by the simulations.

It is now clear how to introduce scaled dimensionless variables $\left(\tilde{t}_{C}\right.$ and $\tilde{t}_{S}$ (with $C=0)$ serving for timescales, $S_{0}$ for both $C$ and $S$ scales in the fast transient, but later $S_{0} K / E_{0}$ for the $S$ scale), in terms of which a singular perturbation analysis 
can be made. But no further calculations are necessary to make the point that our understanding of timescales enabled us to discover a new aspect of the QSSA.

Acknowledgments. Thanks to M.D. Kruskal for reminding us of the Principal of Minimal Simplification, to A. Perelson and A. Goldbeter for improvements on earlier drafts, and to Carol Weintraub for $T_{E} X n i c a l$ brilliance.

\section{REFERENCES}

[1] J. Bowen, A. ACrivos, And A. Oppenheim, Singular perturbation refinement to quasisteady state approximation in chemical kinetics, Chem. Engrg. Sci., 18 (1963), pp. 177-188.

[2] G. E. BRIGgS AND J. B. S. HAldane, A note on the kinetics of enzyme action, Biochem. J. (1925), pp. $338-339$.

[3] N. F. BRITtON, Reaction-diffusion equations and their applications to biology, Academic Press, London, 1986.

[4] J. CARR, Applications of Center Manifold Theory, Springer-Verlag, New York, 1981.

[5] G. F. CARRIER, Singular perturbation theory and geophysics, SIAM Rev., 12 (1970), pp. $175-93$.

[6] E. Coddington And N. Levinson, Theory of Ordinary Differential Equations, McGraw Hill, New York, 1955, pp. 3-7.

[7] G. DAHLQUIST, On transformations at graded matrices with applications to stiff ODE's, Numer. Math., 47 (1985), pp. 363-385.

[8] G. Dahlquist, L. Edsberg, G. Sköllermo, and G. Söderlind, Are the numerical methods and software satisfactory for chemical kinetics? Numerical Integration of Differential Equations, J. Hunze, ed., Springer-Verlag, Berlin, 1980.

[9] N. De Bruijn, Asymptotic Methods in Analysis, North-Holland, Amsterdam, 1970.

[10] W. ECKhaus, Matched Asymptotic Expansions and Singular Perturbations, North-Holland, Amsterdam, 1973.

[11] A. FERSht, Enzyme Structure and Mechanism, Second edition, W.F. Freeman, New York, 1985.

[12] C. L. FRENZEN AND P. K. MAINI, Enzyme kinetics for a two-step enzymic reaction with comparable initial enzyme-substrate ratios, J. Math. Biol., 26 (1989), pp. 689-703.

[13] H. HAHN, Geometrical aspects of the pseudo steady state hypothesis in enzyme reactions, in Physics and Mathematics of the Nervous System, M. Conrad, W. Guttinger, and M. Dal Cin, eds., Springer-Verlag, Berlin, 1974, pp. 528-546.

[14] F. HEINEKEN, H. TSUCHIYA, AND R. ARIS, On the mathematical status of the pseudo-steady state hypothesis of biochemical kinetics, Math. Biosci., 1 (1967), pp. 95-113.

[15] V. HENRI, Lois générales de l'action des diastases, Paris, Hermann, 1903.

[16] W. KLONOWSKI, Simplifying principles for chemical and enzyme reaction kinetics, Biophys. Chem., 18 (1983), pp. 7387.

[17] M. D. KRUSKal, Asymptotology, in Mathematical Models in Physical Sciences, S. Drobot, ed., Prentice-Hall, Englewood Cliffs, NJ., 1963, pp. 17-48.

[18] K. LAIDLER, Theory of the transient phase in kinetics, with special reference to enzyme systems, Canad. J. Chem., 33 (1955), pp. 1614-1624.

[19] A. Lehninger, Principles of Biochemistry, Worth, New York, 1982.

[20] C. C. Lin And L.A. Segel, Mathematics Applied to Deterministic Problems in the Natural Sciences, MacMillan, New York, 1974. Reprinted by the Society for Industrial and Applied Mathematics, Philadelphia, 1988.

[21] L. Michaelis AND M. L. MENTEN, Die Kinetik der Invertinwirkung, Biochem. Z., 49 (1913), pp. 33369 .

[22] J. D. Murray, Lectures on Nonlinear-Differential-Equation Models in Biology, Clarendon Press, London, 1977.

[23] K. NIPP, A formal method for matched asymptotic expansions applied to the Field-Noyes model of the Belousov Zhabotinskii reaction, Mech. Res. Comm., 5 (1978), pp. 231-238.

[24] —, An algorithmic approach to singular perturbation problems in ordinary differential equations with an application to the Belousov-Zhabotinskii reaction, Ph.D. dissertation ETH6643, Swiss Federal Institute of Technology, Zurich, 1980.

[25] G. M. ODELL AND L.A. SEgEL, BIOGRAPH. A graphical simulation package with exercises to accompany Lee A. Segel's "Modeling Dynamic Phenomenon in Molecular and Cellular Biology", Cambridge University Press, Cambridge, 1987. 
[26] H. Parnas, M. Flashner and M. Spira, A sequential model to describe the nicotinic synaptic current. Biophys. J., in press.

[27] J. REICH AND E. SEL'KOV, Mathematical analysis of metabolic networks, FEBS Lett., 40S, (1976) pp. 119-127.

[28] S. RUBinow, Introduction to Mathematical Biology, John Wiley, New York, 1975.

[29] M. SChaUer AND R. HeinRICH, Analysis of the quasi-steady-state approximation for an enzymatic one-substrate reaction, J. Theoret. Biol., 79 (1979), pp. 425-442.

[30] L. A. SEGEL, Simplification and scaling, SIAM Rev., 14 (1972), pp. 547-571.

[31] — Modeling Dynamic Phenomena in Molecular and Cellular Biology, Cambridge University Press, Cambridge, 1984.

[32] - On the validity of the steady state assumption of chemical kinetics, Bull. Math. Biol., 50 (1988), pp. 579-593.

[33] M. SeshadRI AND G. FRITzSCH, Analytical solutions of a simple enzyme kinetic problem by a perturbative procedure, Biophys. Struct. Mech., 6 (1980), pp. 111-123.

[34] A. SOLS AND R. MARCO, Concentrations of metabolites and binding sites. Implications in metabolic regulation, in Current Topics in Cellular Regulation, Vol. 2, B. Horecker and E. Stadtman, eds., Academic Press, New York, 1970, pp. 227-273.

[35] M. STAYTON AND H. FROMm, A computer analysis of the validity of the integrated MichaelisMenten equation, J. Theoret. Biol., 78 (1979), pp. 309-323.

[36] J. T.-F. WONG, On the steady-state method of enzyme kinetics, J. Amer. Chem. Soc., 87 (1964), pp. $1788-1793$.

[37] M. ZU-FAN, Partioning a stiff ordinary differential system by a scaling technique, Report TRITA-NA-8210, Department of Numerical Analysis and Computer Science, Royal Institute of Technology, S-100 44 Stockholm 70, Sweden, 1982. 